\title{
Expressive Synchronization Types for Inheritance in the Join Calculus
}

\author{
Qin Ma Luc Maranget
}

$\mathbf{N}^{\circ} 4889$

July 21, 2003

THÈME 1 



\title{
Expressive Synchronization Types for Inheritance in the Join Calculus
}

\author{
Qin Ma Luc Maranget \\ Thème 1 - Réseaux et systèmes \\ Projet Moscova \\ Rapport de recherche $\mathrm{n}^{\circ} 4889$ - July 21,2003 - 41 pages
}

\begin{abstract}
In prior work, Fournet et al. proposed an extension of the join calculus with class-based inheritance, aiming to provide a precise semantics for concurrent objects. However, as we show here, their system suffers from several limitations, which make it inadequate to form the basis of a practical implementation.

In this paper, we redesign the static semantics for inheritance in the join calculus, equipping class types with more precise information. Compared to previous work, the new type system is more powerful, more expressive and simpler. Additionally, one runtime check of the old system is suppressed in the new design. We also prove the soundness of the new system, and have implemented type inference.
\end{abstract}

Key-words: concurrency, object-oriented, programming language, type system, join calculus, class-based inheritance 


\section{Types expressifs pour l'héritage dans le join-calcul}

Résumé : Nous poursuivons ici les travaux de Fournet et al. sur une extension orientée objet du join-calcul. De notre point de vue, Fournet et al. donnent à la fois une sémantique précise aux objets concurrents et une conception saine de la programmation incrémentale à base d'héritage de classe dans un contexte concurrent. Cependant, comme nous le montrons ici, leur système souffre de plusieurs limitations et, en pratique, on ne peut pas envisager son utilisation tel quel dans une implémentation.

En rendant les types des classes plus informatifs, nous proposons ici un nouveau système dérivé du précédent. Le nouveau système de types se révèle plus puissant, plus expressifs et plus simple à comprendre par le programmeur qui devrait pouvoir plus facilement corriger ses erreurs au vu des messages du compilateur. En outre, le nouveau système se passe d'une vérification de type dynamique nécessaire dans l'ancien système.

Nous prouvons ici la sûreté du nouveau système et avons par ailleurs mis en œuvre l'inférence de types.

Mots-clés : concurrence, orienté-objet, langages de programmation, systèmes de types, join-calcul, héritage bassé sur la classe 


\section{Introduction}

Object-oriented programming is an attractive framework for taming the complexity of modern concurrent systems. In the standard approach, as in Java [11], ABCL/1 [18] or Obliq [4], objects represent agents that may exchange messages over a network. These languages usually model concurrency by threads and control synchronization behavior with locks (or some variant thereof). However, combining concurrency and object-oriented programming as if they were independent hinders incremental code development by inheritance [13]. Roughly, objects follow synchronization policies to keep their internal state consistent in the presence of concurrent method calls, but traditional inheritance is not sufficient to program these policies incrementally.

In [9], Fournet et al. address this issue. They define the objective-join calculus, aiming to provide a precise semantics for concurrent objects. Doing so, they follow many authors, who either enrich well-established object calculi with concurrency primitives $[10,2,14]$, or extend process calculi with object-oriented features [17]. The base calculus of [9] is the join calculus [7], a simple name-passing calculus, related to the pi-calculus but with a functional flavor. Objects are introduced by the classical technique of objects-as-records (of names). This technique is particularly natural in the join calculus, where several names and their behavior with respect to synchronization are defined simultaneously, via join-definitions. Then, [9] introduces a class layer to support incremental programming and code reuse.

However, there are several limitations to the theory presented in [9] which make it inadequate to form the basis of a practical implementation. The present paper overcomes these limitations, focusing on the typing of the class layer. Class types of [9] are close to their sequential counterparts: class types list names with the types of the messages they accept. This information is sufficient for typing objects, whose basic operation is message receiving, but is too limited for typing class-based inheritance of synchronization behavior. As a consequence, the class types of [9] in fact include some extra information about synchronization behavior. But this information is not expressive enough, leading to the following shortcomings: objects sometimes are not as polymorphic as they could; names flagged as abstract by typing may actually possess a definition; and, under particular conditions, a complicated runtime check on classes is necessary to guarantee program safety. We solve these difficulties with a new type system for classes, of which the main contribution is the inclusion of complete synchronization behavior in class types.

The paper is organized as follows. We give a brief review of the object and class calculus in Sec. 2. Then, from Sec. 3 to Sec. 5, we point out the shortcomings of

$\mathrm{RR} \mathrm{n}^{\circ} 4889$ 
the former system by means of examples. Sec. 6 presents the new design for the static class semantics and states the properties of subject reduction and type safety though omits the lengthy proofs (given in an appendix). Finally, we conclude.

\section{A brief review of the objective-join calculus}

\subsection{Objects and classes}

Objects arise naturally in the join calculus when the join-definitions are named and lifted to be the values of the calculus. For instance, a one-place buffer object is defined as follows:

$$
\begin{aligned}
& \text { obj buffer }= \\
& \quad \text { put }(n, r) \& \operatorname{Empty}() \triangleright \text { r.reply }() \text { \& buffer.Some }(n) \\
& \text { or get }(r) \& \operatorname{Some}(n) \triangleright r . r e p l y(n) \& \text { buffer.Empty }() \\
& \text { init buffer.Empty }()
\end{aligned}
$$

The basic operation is still asynchronous message passing, but expressed by objectoriented dot notation. Here, the process buffer.put $(n, r)$ sends a put $(n, r)$ message to the object buffer. In the message, put is the label and $(n, r)$ is the content.

As in join, the labels defined in one object are organized by several reaction rules to specify how messages sent on these labels will be synchronized and processed. In the above buffer object, four labels are defined and arranged in two reaction rules. Each reaction rule consists of a join-pattern and a guarded process, separated by $D$. When there are messages pending on all the labels in a given pattern, the object can react by consuming the messages and triggering the guarded process. Given this synchronization mechanism, buffer will behave as follows:

- If buffer is empty (i.e. an Empty() message is pending), and a put attempt is made (by sending a put $(n, r)$ message), then buffer will react by sending back an acknowledgement message on label reply of the continuation $r$ and, concurrently, shifting itself into the Some $(n)$ state.

- Symmetrically, the value $n$ stored in buffer (i.e. a Some ( $n$ ) message is pending) can be retrieved by $g e t(r)$, and the buffer then returns to the empty state.

- Any put requests sent to a full buffer (or get request to an empty buffer) will be delayed until the object is changed into the complementary state by other messages. 
Finally, the (optional) init part initializes buffer as an empty buffer. See [1] for a more implementation-oriented description of a similar example.

As we can see, the state of buffer is encoded as a message pending on Empty or on Some. To keep the one-place buffer consistent, there should be only one message pending on either of these two labels. This invariant holds as long as no message on these labels can be sent from outside, which can be guaranteed by the following a statically enforced privacy policy: Labels starting with an uppercase letter are private labels; messages can be sent on those labels only from within guarded processes (and from the init part as well). Other labels are unrestricted; they are called public labels and work as ports offered by the object to the external world.

Classes act as templates for sets of objects with the same behavior. For instance, the class defining the one-place buffer above looks like this:

$$
\begin{aligned}
& \text { class } c_{-} \text {buffer }=\operatorname{self}(z) \\
& \quad \operatorname{get}(r) \& \operatorname{Some}(n) \triangleright r . r e p l y(n) \& \text { z.Empty }() \\
& \text { or put }(n, r) \& \text { Empty }() \triangleright \text { r.reply }() \& \text { z.Some }(n)
\end{aligned}
$$

The prefix self $(z)$ explicitly binds the name $z$ to the self reference in the class definition. And to instantiate an object from the class, we just do:

obj buffer $=c_{-}$buffer init buffer.Empty ()

The calculus of [9] allows the derivation of new class definitions from existing ones. A class can be extended with new reaction rules, synchronizations among the labels of a class can be modified, and new processes can be added to the reaction rules of a class. These computations on classes finally yield classes solely made of disjunctions (or) of several reaction rules (as is the $c_{-} b u f f e r$ class). Such classes are ready for being instantiated and are called normal forms.

\section{$2.2 \quad$ Types}

Types for classes and objects are inspired by the OCaml type system [16]. More precisely, typing is by structure; both class types and object types are sets of label names paired with the types of the messages they accept; object types only collect public labels, while class types include both private and public labels. Object types may end with a row variable, standing for more label-type pairs and such row variables provide a useful degree of polymorphism.

For instance, consider a simple synchronization object join with an internal counter:

obj join $=\operatorname{sync1}(r 1) \& \operatorname{sync2}(r \mathscr{2}) \& \operatorname{Count}(x) \triangleright$

$\mathrm{RR} \mathrm{n}^{\circ} 4889$ 


\section{r1.reply() \& r2.reply () \& join.Count $(x+1)$}

The type of the object join is $[$ sync1 : ([reply: ()$; \varrho]) ;$ sync2 : ([reply : ()$\left.\left.\left.; \varrho^{\prime}\right]\right)\right]$. The two row variables $\varrho$ and $\varrho^{\prime}$ indicate that except for the reply label, there is no other constraints on the continuation objects sent to the sync labels. Furthermore, label Count does not show up because it is private. By contrast, the type of the corresponding $c_{-}$join class contains all the labels:

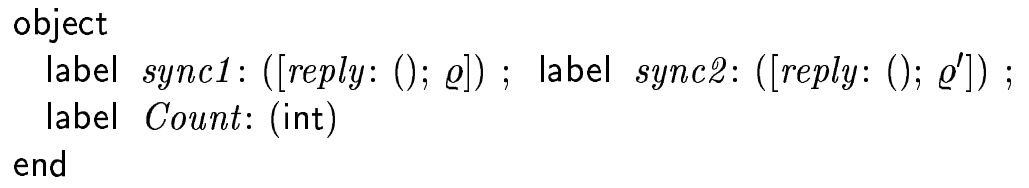

However, during the design of the type system of [9], it appeared that class types should include additional information. We introduce them in the next two sections using examples.

\section{Polymorphism}

\subsection{Polymorphism and synchronization}

There are two kinds of type variables in the type system: ordinary type variables and row variables. As in the ML type system, polymorphism is parametric polymorphism, obtained essentially by generalizing the free type variables.

As in the join calculus, synchronizations impact on polymorphism [8, 5]: two different labels are said to be correlated when they appear in the same join-pattern, and type variables that appear in the types of several correlated labels cannot be generalized. Consider, for instance, a synchronous buffer object:

obj sbuffer $=\operatorname{get}(r) \& \operatorname{put}(n, s) \triangleright$ r.reply $(n) \&$ s.reply ()

The types of the two correlated labels get and put are $([$ reply $:(\theta) ; \varrho])$ and $(\theta,[$ reply: ()$\left.\left.; \varrho^{\prime}\right]\right)$, respectively. Type variable $\theta$ is shared by their types, so it should not be generalized. Otherwise, the two occurrence of $\theta$ could be instantiated independently as, for instance, integer and string. This then might result in a runtime type error: attempting to deliver a string when an integer is expected. By contrast, the type variables $\varrho$ and $\varrho^{\prime}$ can be generalized. Generalized row variable $\varrho$ intuitively means that as long as the constraint on reply label is preserved, different messages sent on label get can take different continuation objects as $r$, and similarly for $\varrho^{\prime}$ with respect to label put.

However, the limitation on polymorphism is only object-level, not class-level, because two get or put labels of two different objects are completely independent 
w.r.t. synchronization. More concretely, consider the following class version of the synchronous buffer.

$$
\text { class } \quad c \_s b u f f e r=\operatorname{get}(r) \& \operatorname{put}(n, s) \triangleright r \text { reply }(n) \& \text { s.reply }()
$$

Class $c_{\_} s b u f f e r$ can be instantiated twice to two different objects, one dealing with integers, and the other with strings. As a consequence, all the free type variables in class types are generalized, but class types record the correlation amongst labels, in order to restrict polymorphism at object creation time. To collect this information, the type system of [9] abstracts the join-patterns of a class into a set of coupled labels $W$. The computation of coupled labels from reaction rules can be described as a two-phase process:

1. First, simplify join-patterns by removing labels accepting empty tuple (because correlating with this kind of labels does not cause type variable sharing) and remove join-patterns consisting of only one label.

2. Then, gather all the remaining labels into the set of coupled labels.

The set $W$ is then kept in the class type, and referred to when an object is to be created: all the type variables shared by several labels in $W$ are restricted to be monomorphic in the corresponding object type. As a result, the type of the synchronous buffer class is as follows:

$$
\begin{gathered}
\text { class c_sbuffer: } \forall \theta, \varrho, \varrho^{\prime} \text {. object } \\
\text { label get: }([\text { reply: }(\theta) ; \varrho]) ; \\
\text { label put: }\left(\theta,\left[\text { reply: }() ; \varrho^{\prime}\right]\right) \\
\text { end } W=\{\text { get }, \text { put }\}
\end{gathered}
$$

Here labels get and put are coupled, and hence the type variable $\theta$ is not generalizable in object types.

\subsection{More polymorphism in object types}

According to the previous section, the following object definition:

obj counter $=$

$\operatorname{Value}(x) \& \operatorname{plus}(y, r) \triangleright r$.reply ()$\&$ counter.Value $(x+y)$

or Value $(x) \& \operatorname{incr}(r) \triangleright$ counter.Value $(x) \&$ counter.plus $(1, r)$

correlates the labels plus and Value by the first join-pattern, and the labels incr and Value by the second join-pattern (but not the labels plus and incr). The types of the labels of this object are Value: (int), plus: (int, $[$ reply : (); $]$ ) and incr:

$\mathrm{RR} \mathrm{n}^{\circ} 4889$ 
$([$ reply : ()$; \varrho])$. The free row variable $\varrho$ appears in the types of both plus and incr, but since the two labels are not correlated, it can be generalized.

However, lifting the object definition counter into a class definition will yield the class $c_{-}$counter with type:

class c_counter: object... end $W=\{$ Value, plus, incr $\}$

Both plus and incr appear as coupled labels, which forbids the generalization of the shared free type variable $\varrho$ in the type of the objects.

This undue restriction on polymorphism originates in the flat structure of the set $W$. Such a structure may couple labels that are not actually correlated, more precisely, the coupled label relation is the transitive closure of correlation amongst labels accepting non-empty tuples. In the counter example, labels plus and incr get "correlated" through Value.

However, if we replace the second step of the computation of the set $W$ in Sec. 3.1 by collecting labels from different patterns in separate sets, thereby organizing $W$ as the set of these sets, we may have a better solution. The criterion for restriction changes accordingly: for now, only those type variables shared by two labels coming from the same member set of $W$ are non-generalizable. The new type of the class $c_{-}$counter is

$$
\text { class c_counter: object } \ldots \text { end } W=\{\{\text { Value, plus }\},\{\text { Value, incr }\}\}
$$

We see that the labels plus and incr are separated in different member sets, so that the type variable $\varrho$ can be polymorphic. More generally, the layered structure of the new $W$ gets rid of the transitivity side-effect, and restores proper polymorphism. It is important to notice that the new $W$ sets still abstract on the join-patterns: zero-arity labels and non-synchronized labels do not show up.

\section{Selective refinement and abstract labels}

\subsection{Semantics of selective refinement}

Selective refinement is the only operator on classes that can modify the reaction rules. It can be understood by analogy with ML-style pattern matching. Selective refinement rewrites a class $c$ into a new class $c^{\prime}$ by matching the reaction rules of $c$ against a refinement sequence. A refinement sequence is an ordered list of refinement clauses, and refinement clauses are tried in that order. A refinement clause $K_{1} \Rightarrow K_{2} \triangleright Q$ consists of three parts: the selective pattern $K_{1}$, the refinement pattern $K_{2}$ and the refinement process $Q$. A refinement clause matches a reaction rule $J \triangleright P$ when $K_{1}$ is a sub-pattern of $J$, that is, when all the labels in $K_{1}$ also 
appear in $J$. Then, $J$ can be written as $K_{1} \& K$ and the matched reaction rule is refined into the new reaction $K \& K_{2} \triangleright P \& Q$. As a consequence, a reaction rule is rewritten according to the first matching refinement clause if it exists. Otherwise, the reaction rule remains unchanged.

\subsection{Abstract labels after selective refinement}

Now let us consider again the class $c_{-}$counter of Sec. 3.2 and assume that, for debugging purpose, we wish to log each plus attempt on the terminal. This can be achieved by a two-step modification: First, the plus label is renamed into unlogged_plus, using selective refinement; then, the plus label is redefined:

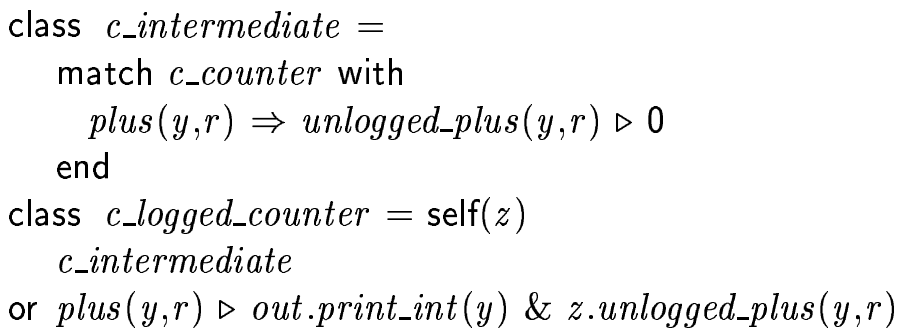

The type of the class $c \_i n t e r m e d i a t e$ is as follows:

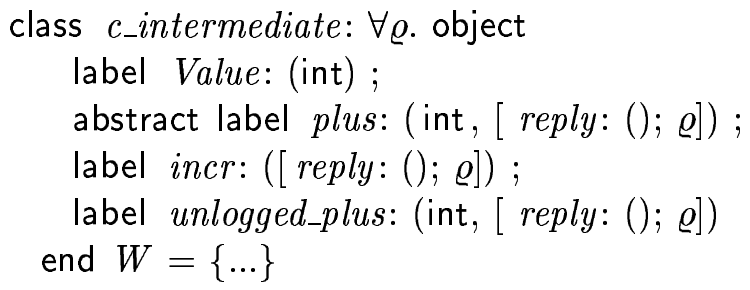

It is important to notice that this type is inferred at compile time, considering only the type of the class $c_{-}$counter and the refinement clauses, without actually performing the selective refinement. We observe that the label plus is still present in the type of class c_intermediate. However, this label is now tagged as abstract. In fact, the normal form of class $c_{-}$intermediate as computed at runtime looks like:

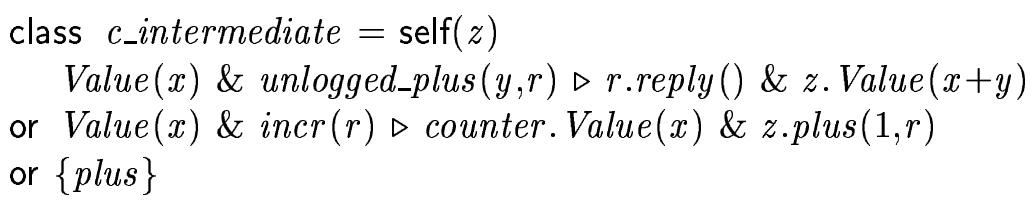

The label plus does not appear in the patterns any more, but it still appears in the guarded process of the second reaction rule. This fact is accounted for by stating that plus is abstract in class $c_{-}$intermediate, using the new construct $\{$plus $\}$. As in the

$\mathrm{RR} \mathrm{n}^{\circ} 4889$ 
ordinary sense of object-oriented programming, classes with abstract labels cannot be instantiated. Therefore, class $c_{-}$intermediate is prevented from instantiation until a concrete and type compatible definition for plus is provided, as performed by the definition of class c_logged_counter. This constraint is reflected by the abstract tag in the class type.

\subsection{Abstract or maybe abstract}

We move on to another example: a machine creator (the class) which creates vendor machines (the objects) selling both tea and coffee.

$$
\begin{aligned}
& \text { class } c_{-} v e n d o r= \\
& \text { two_euros }() \text { \& tea }() \triangleright \text { out.print_string("tea") } \\
& \text { or two_euros }() \text { \& coffee }() \triangleright \text { out.print_string("coffee") }
\end{aligned}
$$

Later, the price of tea decreases to one euro, so the machine creator should be modified. Such a modification is attempted by selective refinement:

$$
\begin{aligned}
& \text { class } c_{-} \text {promo_vendor }= \\
& \text { match } c_{-} \text {vendor with } \\
& \left.\quad \text { two_euros }() \text { \& tea }() \Rightarrow \text { one_euro }_{(}\right) \text {\& tea }() \triangleright 0 \\
& \text { end }
\end{aligned}
$$

The normal form of class c_promo_vendor at runtime is:

$$
\begin{aligned}
& \text { class } c_{-} \text {promo_vendor }= \\
& \text { one_euro }() \text { \& tea }() \triangleright \text { out.print_string( "tea") } \\
& \text { or two_euros }() \text { \& coffee }() \triangleright \text { out.print_string("coffee") }
\end{aligned}
$$

Unfortunately, the type of class c_promo_vendor tags two_euros as abstract:

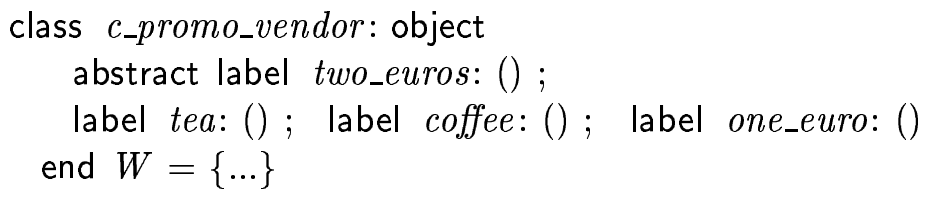

And this will, in practice, prevent from creating any real new vendor machine objects from this class.

The discrepancy between the compile time type and the runtime normal form comes from the way selective refinement is typed. Since types do not include all synchronization information, the effect of selective refinement can only be approximated while typing. As regards labels that no more exist in the join-patterns of the resulting class, the approximation can be described as follows: 
1. For each refinement clause $K_{1} \Rightarrow K_{2} \triangleright Q$, compute the set of labels that appear in $K_{1}$ but not in $K_{2}$.

2. Take the union of the previous sets of all clauses in the selective refinement.

As demonstrated by the refinement from $c_{-}$vendor to $c_{-} p r o m o \_v e n d o r$, this approximation is not always exact. As an untimely consequence, the abstract qualifier in types significantly departs from its usual meaning: an abstract label is in fact a label that may lack a definition, while common sense and tradition suggest that abstract labels should be labels with no definition. Accordingly, the information conveyed by the abstract qualifier in types is significantly reduced.

This abstract as maybe abstract semantics is expressed by a "subsumption" rule Sub in the type system of [9]. Basically, this rule lifts abstract label set inclusion into subtyping. Such a subtyping is of little practical interest.

However, if selective refinement can be effectively carried out during typing, the type of compile time will be able to tell exactly which label is abstract in the runtime normal form. Then the expedient of the Sub rule can be withdrawn. It is certainly a more practical solution compared with the former type system.

\section{$5 \quad$ New labels during selective refinement}

In the previous section, we saw that some labels in a class can lose their definitions during selective refinement. Symmetrically, selective refinement introduces new labels. Those new labels endanger the basic type safety property of avoiding "message not understood" errors. For instance, consider the following selective refinement.

$$
\text { class } c c=\text { match } c \text { with } x() \& y() \Rightarrow z() \triangleright 0 \text { end }
$$

We additionally assume that class $c$ indeed possesses the two labels $x$ and $y$, as shown by its type.

$$
\text { class } c \text { : object label } x:() \text {; label } y:() \text { end } W=\{\ldots\}
$$

Then, label $z$ is new to the class $c$, and by the typing of [9], the type of the resulting class $c c$ will include the new label:

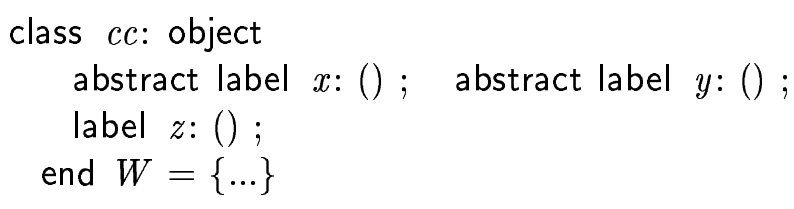

However, class $c$ can be either of the following two definitions, because both of them have the type of class $c$ given above.

$\mathrm{RR} \mathrm{n}^{\circ} 4889$ 


$$
\begin{aligned}
& \text { class } c_{1}=x() \& y() \triangleright P_{1} \\
& \text { class } c_{2}=x() \triangleright P_{2} \text { or } y() \triangleright Q_{2}
\end{aligned}
$$

If $c$ is $c_{1}$, the runtime normal form of class $c c$ will effectively possess the new label $z$. But, if $x$ and $y$ are not synchronized (i.e. if $c$ is $c_{2}$ ), then class $c c$ does not provide a definition for label $z$. Obviously, if nothing is done for the latter case, basic type safety is broken. The solution adopted in [9] enforces the actual introduction of all new labels. In practice, a runtime check of the presence of some labels (in this example, label $z$ ) has to be performed after selective refinement. When some labels are missing, the running program fails with a specific refinement error. From a language design perspective, this solution is moderately acceptable.

1. It can be argued that programmers would design selective refinements with the intention to define some new labels.

2. Refinement error is by far more tolerable than the "message not understood" error. The former may only show up when building classes, while the latter may occur when using objects. Hence, refinement error occurs earlier, less frequently, and conveys properer information for easier correctness by programmers.

However, guaranteeing type safety at no runtime price is certainly a better solution. This can be achieved provided types are detailed enough, so that pattern matching on reaction rules can be performed while typing. Then, only effectively introduced new labels show up in the resulting type.

\section{The new type system}

Based on the discussions in the previous three sections, we present our new type system. Our type system elaborates on the one of [9]. The main novelty lies in equipping each class type with the structure of all its join-patterns, but there are other differences. In our description, we mostly point out changes w.r.t. the former system. We do not include here the syntax of objects and classes, nor their semantics, since those are unchanged except for the suppression of one runtime check (see Sec. 5).

\subsection{Type algebra}

The type algebra of the new type system appears in Fig. 1. The class types are written as $\zeta(\rho) B^{W}$. As before, $B$ lists the types of all the declared labels in the 


$$
\begin{aligned}
\tau::=\theta \mid[\rho] & \text { Object type } \\
\rho::=\emptyset|\varrho| m: \widetilde{\tau} ; \rho & \text { Row type } \\
\sigma::=\forall X . \tau & \text { Type scheme } \\
\alpha::=\theta \mid \varrho & \text { Type Variable } \\
\widetilde{\tau}::=\left(\tau_{i} i \in I\right) & \text { Tuple type } \\
B::=\emptyset \mid \ell: \widetilde{\tau} ; B & \text { Internal type } \\
\tau^{c}::=\zeta(\rho) B^{W} & \text { Class type } \\
\sigma^{c}::=\forall X . \tau^{c} & \text { Class type scheme } \\
\tau^{s}::=B F & \\
F::=\emptyset \mid \pi \Rightarrow W ; F & \text { Refinement sequence type } \\
F^{F} & \text { Refinement rules }
\end{aligned}
$$

Figure 1: Syntax for the type algebra

class and $[\rho]$ refers to the object type of self. By contrast, our correlated label set $W$ replaces the coupled label set of [9]. Following the two-layer structure introduced in Sec. 3.2, $W$ is a set of sets of labels, namely, $W \subseteq 2^{\mathcal{L}}(\mathcal{L}$ collects all the labels in this calculus). Member sets of $W$ are ranged over by $\pi(\pi \subseteq \mathcal{L})$, each $\pi$ corresponding to one join-pattern, and the whole set $W$ represents all the patterns of the class normal form. Our class types do not include a set of abstract labels, while the types of [9] did. The set of abstract labels can now be computed easily from class types as $\operatorname{dom}(B) \backslash \bar{W}$, where $\bar{W}$ is the flattening of $W$ (union of all member sets) and $\operatorname{dom}(B)$ is the domain of $B$. Moreover, by contrast, the abstract labels computed in this manner are abstract in the ordinary sense (see Sec. 4.3)

The type $B^{F}$ of a refinement sequence conveys the latter's whole structure, including refinement clause order. The system of [9] did not attach a particular type to a refinement sequence. Although such a type is not needed to type applications of selective refinement, our solution simplifies the typing rules and reflects our attempt to make selective refinement an autonomous operator. 


\subsection{Typing rules}

Our system's typing judgments are as follows:
$A \vdash x: \tau$
object $x$ has type $\tau$ in environment $A$;
$A \vdash x . \ell:: \widetilde{\tau}$
$A \vdash P$
label $\ell$ in object $x$ has type $\widetilde{\tau}$ in environment $A$;
$A \vdash K:: B$ process $P$ is well-typed in environment $A$;
$A \vdash C:: \tau^{c}$ join-pattern $K$ has type $B$ in environment $A$;
$A \vdash S:: \tau^{s}$ class $C$ has type $\tau^{c}$ in environment $A$; refinement sequence $S$ has type $\tau^{s}$ in environment $A$.

Typing judgments rely on type environments $A$ that bind class and object names to type schemes:

$$
A::=\emptyset\left|c: \sigma^{c} ; A\right| x: \sigma ; A \mid x: \forall X . B ; A
$$

An object $x$ may have two complementary bindings in $A, x: \sigma$ (external scheme) for public labels, and $x: \forall X . B$ (internal scheme) for private labels.

The typing rules appear in Fig. 2 and 3. Before we have a close look at the rules, some auxiliary definitions should be mentioned.

- $\left\{\gamma_{\alpha} / \alpha^{\alpha \in X}\right\}$ expresses the substitution of type variables by types.

- $\operatorname{dom}(A)$ is the set of identifiers bound in $A$. $A+A^{\prime}$ equals $\left(A \backslash \operatorname{dom}\left(A^{\prime}\right)\right) \cup A^{\prime}$, where $A \backslash X$ removes from $A$ all the bindings of names in $X$ and $A+x$ : $\forall X .[\rho], x: \forall X . B$ means $A \backslash\{x\} \cup x: \forall X .[\rho] \cup x: \forall X . B$.

- $B \nmid L$ restricts $B$ to the set of labels $L$. Labels $\ell \in \mathcal{L}$ are divided into private labels $f \in \mathcal{F}$ and public labels $m \in \mathcal{M}$.

- $f t v()$ is the set of free type variables occurring in various constructs.

- Gen $(\rho, B, A)$ is the set of the type variables that occur free in row type $\rho$ or in internal type $B$, but not in type environment $A$.

- $\operatorname{ctv}\left(B^{W}\right)$ computes the subset of the free type variables in $B$ that are shared by at least the types of two correlated labels according to $W$.

$$
\begin{aligned}
\operatorname{ctv}\left(B^{W}\right) & =\bigcup_{\pi_{i} \in W} \operatorname{ctv}\left(B^{\left\{\pi_{i}\right\}}\right) \\
\operatorname{ctv}\left(B^{\{\pi\}}\right) & =\bigcup_{l \in \pi, l^{\prime} \in \pi, l \neq l^{\prime}} \operatorname{ftv}(B(l)) \cap \operatorname{ftv}\left(B\left(l^{\prime}\right)\right)
\end{aligned}
$$

- predicate $B_{1} \uparrow B_{2}$ expresses that $B_{1}$ and $B_{2}$ coincide on their common labels. 


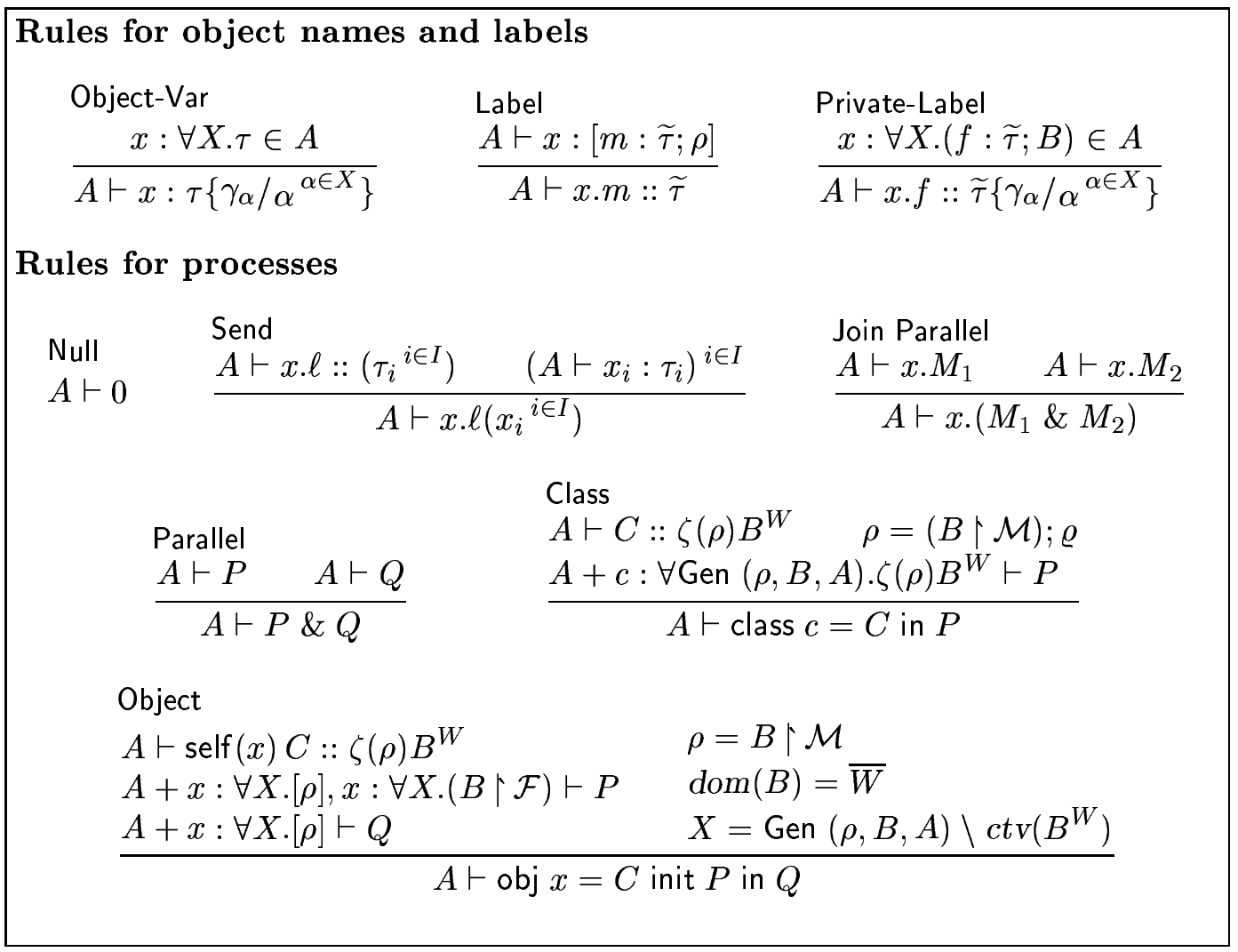

Figure 2: Typing rules for names, and processes

- $B_{1} \oplus B_{2}$ is written for the union of $B_{1}$ and $B_{2}$, provided $B_{1} \uparrow B_{2}$ holds.

- $\operatorname{col}(J)$ computes the correlated labels set from a join-pattern $J$.

$$
\begin{aligned}
\operatorname{col}(\emptyset) & =\emptyset \\
\operatorname{col}(\ell(\widetilde{u})) & =\{\{\ell\}\} \\
\operatorname{col}\left(J \& J^{\prime}\right) & =\left\{\pi_{1} \uplus \pi_{2} \mid \pi_{1} \in \operatorname{col}(J), \pi_{1} \in \operatorname{col}\left(J^{\prime}\right)\right\} \\
\operatorname{col}\left(J \text { or } J^{\prime}\right) & =\operatorname{col}(J) \cup \operatorname{col}\left(J^{\prime}\right)
\end{aligned}
$$

The symbol $\uplus$ means disjoint union, since patterns are required to be linear (or in patterns is a complication, which can be ignored in a first reading).

Processes. The Class rule differs from before by the additional condition $\rho=$ $(B \uparrow \mathcal{M}) ; \varrho$ in the premise. This condition requires an early compatibility check 


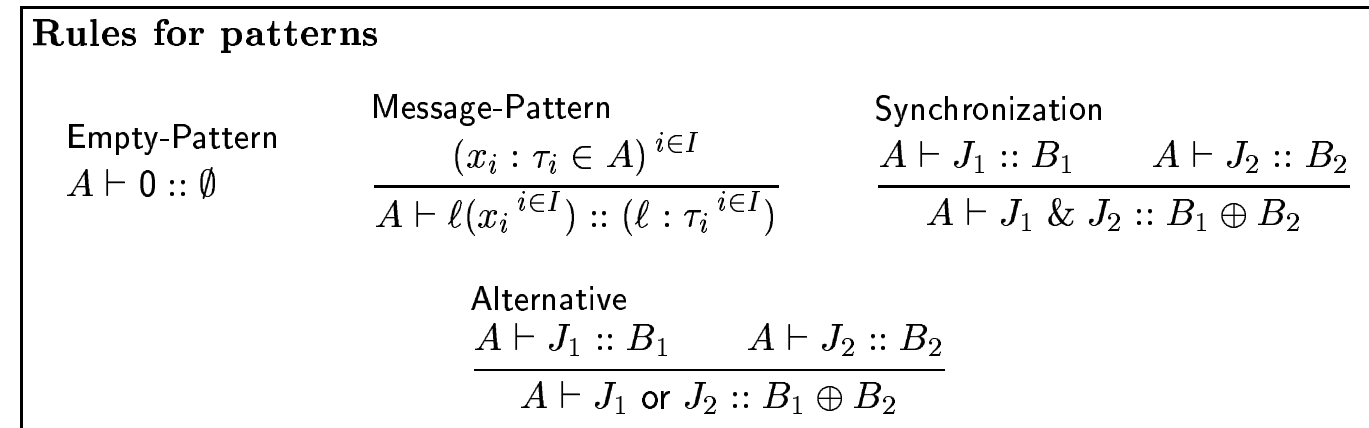

\section{Rules for classes}

$$
\begin{aligned}
& \text { Class-Var } \\
& \text { Self-Binding } \\
& \frac{c: \forall X . \zeta(\rho) B^{W} \in A}{A \vdash c::\left(\zeta(\rho) B^{W}\right)\left\{\gamma_{\alpha} / \alpha^{\alpha \in X}\right\}} \quad \frac{A+x:[\rho], x:(B \nmid \mathcal{F}) \vdash C:: \zeta(\rho) B^{W}}{A \vdash \operatorname{self}(x) C:: \zeta(\rho) B^{W}} \\
& \text { Reaction } \\
& \frac{A^{\prime} \vdash J:: B \quad A+A^{\prime} \vdash P \quad \operatorname{dom}\left(A^{\prime}\right)=f n(J)}{A \vdash J \triangleright P:: \zeta(\rho) B^{\operatorname{col}(J)}} \\
& \text { Abstract } \\
& \operatorname{dom}(B)=L \\
& \overline{A \vdash L:: \zeta(\rho) B^{\emptyset}} \\
& \frac{A \vdash C_{1}:: \zeta(\rho) B_{1}^{W_{1}} \quad A \vdash C_{2}:: \zeta(\rho) B_{2}^{W_{2}}}{A \vdash C_{1} \text { or } C_{2}:: \zeta(\rho)\left(B_{1} \oplus B_{2}\right)^{W_{1} \cup W_{2}}}
\end{aligned}
$$

Refinement

$$
\begin{gathered}
A \vdash C:: \zeta(\rho) B^{W} \\
A \vdash S:: B^{\prime F} \\
\vdash W \text { with } F:: W^{\prime} \quad B \uparrow B^{\prime} \\
\hline A \vdash \text { match } C \text { with } S \text { end }:: \zeta(\rho)\left(\left(B^{\prime} \vdash \overline{W^{\prime}}\right) \oplus B\right)^{W^{\prime}}
\end{gathered}
$$

\section{Rules for refinement clauses}

Modifier-Clause

$$
\begin{array}{cll}
A^{\prime} \vdash K:: B_{1} & A \vdash S:: B^{F} & \\
A^{\prime} \vdash K^{\prime}:: B_{2} & \operatorname{col}(K)=\{\pi\} & \\
A+A^{\prime} \vdash P & \operatorname{dom}\left(A^{\prime}\right)=f n\left(K^{\prime}\right) & \text { Modifier-Em } \\
\hline A \vdash K \Rightarrow K^{\prime} \triangleright P \mid S::\left(B_{1} \oplus B_{2} \oplus B\right)^{\left(\pi \Rightarrow \operatorname{col}\left(K^{\prime}\right)\right) \mid F} & & A \vdash \emptyset:: \emptyset^{\emptyset}
\end{array}
$$

\section{Rules for filters}

$$
\begin{aligned}
& \text { Apply } \\
& \vdash \pi \uplus \pi^{\prime} \text { with } \pi \Rightarrow W \mid F::\left\{\pi^{\prime} \uplus \pi_{i} \mid \pi_{i} \in W\right\} \quad \vdash \pi \text { with } \emptyset::\{\pi\} \\
& \text { Next } \\
& \pi_{1} \not \pi \quad \vdash \pi \text { with } F:: W \\
& \text { Or } \\
& \vdash \pi \text { with } \pi_{1} \Rightarrow W_{2} \mid F:: W \quad \quad \vdash W \text { with } F:: \cup_{\pi_{i} \in W} W_{i}
\end{aligned}
$$

Figure 3: Typing rules for patterns, classes, refinement clauses, and filters 
to verify that the types (in $B$ ) of the public labels inferred from the patterns are compatible with those (in $\rho$ ) inferred from the messages sent to self. In the old system, this check was delayed until object instantiation, which in turn delayed the detection of some errors. Such a delay is particularly annoying in a separate compilation context.

For the Object rule, checking the absence of abstract labels is now performed by the equation $\operatorname{dom}(B)=\bar{W}$. In spite of the new condition on public labels in the Class rule, we retain the condition $\rho=(B \nmid \mathcal{M})$ of [9]. It is still needed for anonymous class definitions, and also has the effect of closing the object type $\rho$. However, the most significant change is the replacement of the imprecise coupled labels by our exact correlated labels in the computation of generalizable type variables.

Classes. All rules are simplified thanks to the disappearance of the explicit abstract labels component in class types. The bizarre Sub rule is not needed any more, as discussed in Sec. 4.3. Our precise correlated labels set is introduced in the rule Reaction, using the external definition $\operatorname{col}(J)$,

The rules for typing selective refinement undergo significant changes. In match $C$ with $S$ end, the typing of class $C$ and the typing of refinement sequence $S$ were combined in [9] but are now independent. Compatibility checks between labels common to $C$ and $S$ are now performed explicitly by condition $B \uparrow B^{\prime}$; furthermore, most of the construction of the resulting type is performed by the auxiliary judgment $W$ with $F:: W^{\prime}$, which computes the patterns of the resulting class. Finally, the effectively introduced new labels (compare to Sec. 5) are added by $B^{\prime} \uparrow \overline{W^{\prime}}$. There is a minor difference w.r.t [9]: our system allows labels in the selective patterns of $S$ that are not necessarily in class $C$. Such a requirement is no longer essential to type safety and we believe that imposing it on programmers is too restrictive. Of course, some warning (analogous to the "unused match case" of ML pattern-matching) can be issued.

Refinement clauses. Rule Modifier-Clause serves both to collect label-type pairs (in $B$ ) and to compute the refinement rules $F$. Processes of refinement clauses $P$ are also typed.

Filters. The rules for typing a filter mimic the semantics of selective refinement, except for the management of abstract names, which is irrelevant. Rules Apply, Next, and End handle the matching of one $\pi$ set by the refinement rule $F$, while rule Or collects the resulting partial $W_{i}$ sets into the resulting correlated label set $W^{\prime}$.

$\mathrm{RR} \mathrm{n}^{\circ} 4889$ 
Linearity of resulting patterns is now checked simply by the disjoint union $\pi^{\prime} \uplus \pi_{i}$ used in the rule Apply. Thereby, we drop one cryptic premise in the Refinement rule of $[9]$.

\subsection{Subject reduction and safety}

The typing is finally extended to chemical solutions in order to illustrate the properties of the type system. An additional judgment: $A \vdash \mathcal{D} \Vdash \mathcal{P}$ is used and the rules are as follows:

$$
\begin{aligned}
& \text { Chemical-Solution } \\
& A=\cup_{\psi x \# D \in \mathcal{D}} A_{x} \\
& \left(A^{\psi} \backslash\{x\} \vdash D:: A_{x}\right)^{\psi x \# D \in \mathcal{D}} \\
& \left(A^{\psi} \vdash P\right)^{\psi \# P \in \mathcal{P}} \\
& \vdash \mathcal{D} \Vdash \mathcal{P} \\
& \text { Definition } \\
& A \vdash \operatorname{self}(x) D:: \zeta(\rho) B^{W} \\
& X=\operatorname{Gen}(\rho, B, A) \backslash \operatorname{ctv}\left(B^{W}\right) \\
& \frac{\rho=B \nmid \mathcal{M} \quad \operatorname{dom}(B)=\bar{W}}{A \vdash D:: x: \forall X .[\rho], x: \forall X .(B \nmid \mathcal{F})}
\end{aligned}
$$

For lack of space, and because this work focuses on type system design, we will not recall the semantics of the objective-join calculus, which can be found in $[9]^{1}$. However, we formally state the correctness of our system.

\section{Theorem 1 (Subject reduction)}

1. Chemical reduction preserves chemical typing:

Suppose $\vdash \mathcal{D} \Vdash \mathcal{P}$. If $\mathcal{D} \Vdash \mathcal{P} \equiv \mathcal{D}^{\prime} \Vdash \mathcal{P}^{\prime}$ or $\mathcal{D} \Vdash \mathcal{P} \rightarrow \mathcal{D}^{\prime} \Vdash \mathcal{P}^{\prime}$, then $\vdash \mathcal{D}^{\prime} \Vdash \mathcal{P}^{\prime}$.

2. Class rewriting preserves typing: if $A \vdash P$ and $P \longmapsto P^{\prime}$ then $A \vdash P^{\prime}$.

Theorem 2 (Safety) Well-typed chemical solutions do not fail.

Refer to Appendix A for the definition of failures and to Appendix B for proofs.

\footnotetext{
${ }^{1}$ In the class semantics, the premise $d l(S) \subseteq d l\left(C^{\prime}\right)$ can be erased from rule Match
} 


\section{Conclusion and future work}

Class types, also known as class interfaces or class signatures, should characterize the externally visible properties of classes. We claim that when concurrent objects are concerned, the synchronization behavior is one such critical property. Based on this idea, we redesigned the static semantics of the concurrent object calculus of [9], equipped class types with their join patterns, and came up with a simpler but more expressive type system. This new type system has more polymorphism and accepts more programs. Furthermore, it is easier for programmers to understand and use, and suppresses the runtime overhead that was essential to safety in the old system. In addition, we also implemented type inference adapting some of the efficient techniques from [15].

Future work. As putting whole join-patterns into class types compromises abstraction, whether and how we can regain it is an interesting question. We see that the current type system handles all class operations (instantiation, disjunction and selective refinement). If we impose some restrictions on the application of certain operators to certain classes, class types can then be more abstract. For example, when some class is tagged as final, the only required synchronization-related information is the set of non-generalizable type variables. So instead of keeping the join-patterns, we can record just this set of variables. More generally, different restrictions will require different amount of synchronization information, and we can eventually reach a multi-leveled framework of abstracting class types, in which a class type may be assigned different degrees of abstraction in different contexts.

Another way to introduce abstraction is by hiding labels defined in a class. Such labels do not show up in the class type, so are untouched by later inheritance and overriding. This will in turn cause significant changes in the semantics of the class operations, probably leading us to a more powerful calculus.

In the old type system [9], selective refinement is typed per application. It would be more interesting if we could make selective refinement an autonomous class-toclass operator. Giving each selective refinement an independent type as we have done in our current type system falls short of this goal: the description of the parameters accepted is missing, and it is impossible to infer the resulting class by looking at the type of selective refinement alone. We wish to integrate our work with current research on mixins $[3,6]$ and hopefully make the selective refinement a mixin-like operator in the concurrent setting.

$\mathrm{RR} \mathrm{n}^{\circ} 4889$ 
Our ultimate goal is to implement our object-oriented extension of join calculus as a concurrent object-oriented programming language. Since the JoCaml [12] implementation already incorporates the join calculus into OCaml, we will pursue our goal by extending JoCaml.

Acknowledgements. The authors wish to thank James Leifer and Pierre-Louis Curien for their comments on drafts of this paper.

\section{References}

[1] N. Benton, L. Cardelli, and C. Fournet. Modern concurrency abstractions for $C^{\#}$. In Proceedings of ECOOP 2002, LNCS 2374, pages 415-440, June 2002.

[2] P. D. Blasio and K. Fisher. A calculus for concurrent objects. In Proceedings of CONCUR '96, LNCS 1119, pages 655-670, Aug. 1996.

[3] G. Bracha and W. Cook. Mixin-Based Inheritance. In Proceedings of OOPSLA.ECOOP '90, pages 303-311. ACM press, Oct. 1990.

[4] L. Cardelli. Obliq: A language with distributed scope. Computing Systems, 8(1):27-59, 1995.

[5] G. Chen, M. Odersky, C. Zenger, and M. Zenger. A functional view of join. Technical Report ACRC-99-016, University of South Australia, 1999.

[6] M. Flatt, S. Krishnamurthi, and M. Felleisen. Classes and mixins. In Proceedings of POPL '98, pages 171-183. ACM press, Jan. 1998.

[7] C. Fournet and G. Gonthier. The reflexive chemical abstract machine and the join-calculus. In Proceedings of POPL '96, pages 372-385. ACM press, Jan. 1996.

[8] C. Fournet, C. Laneve, L. Maranget, and D. Rémy. Implicit typing à la ML for the join-calculus. In Proceedings of CONCUR '97, LNCS 1243, pages 196-212, July 1997.

[9] C. Fournet, L. Maranget, C. Laneve, and D. Rémy. Inheritance in the join calculus. Journal of Logic and Algebraic Programming. To appear, http://

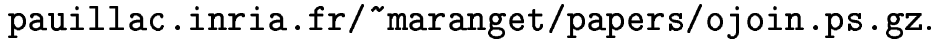


[10] A. D. Gordon and P. D. Hankin. A concurrent object calculus: reduction and typing. In Proceedings of HLCL '98, ENTCS 16(3), Sept. 1998.

[11] B. J. James Gosling and G. Steele. The Java Language Specification. Addison Wesley Longman Inc., Reading, Mass., 1996.

[12] F. Le Fessant. The JoCaml system prototype. Software and documentation available from http://pauillac.inria.fr/jocaml, 1998.

[13] S. Matsuoka and A. Yonezawa. Analysis of inheritance anomaly in objectoriented concurrent programming languages. In G. Agha, P. Wegner, and A. Yonezawa, editors, Research Directions in Concurrent Object-Oriented Programming, chapter 4, pages 107-150. The MIT Press, 1993.

[14] O. Nierstrasz. Towards an object calculus. In Proceedings of ECOOP '91 Workshop on Object-Based Concurrent Computing, LNCS 612, pages 1-20, 1992.

[15] D. Rémy. Extension of ML type system with a sorted equation theory on types. Rapport de recherche 1766, INRIA-Rocquencourt, BP 105, F-78153 Le Chesnay Cedex, France, Oct. 1992.

[16] D. Rémy and J. Vouillon. Objective ML: An effective object-oriented extension to ML. Theory And Practice of Object Systems, 4(1):27-50, 1998.

[17] V. T. Vasconcelos. Typed concurrent objects. In Proceedings of ECOOP '94 Workshop on Object-Based Concurrent Computing, LNCS 821, pages 100-117, July 1994.

[18] A. Yonezawa, J.-P. Briot, and E. Shibayama. Object-oriented concurrent programming in ABCL/1. In Proceedings of OOPSLA '86, ACM SIGPLAN Notices 21(11), pages 258-268, Nov. 1986.

$\mathrm{RR} \mathrm{n}^{\circ} 4889$ 


\section{A Definitions for failures}

Definition 1 (Class rewriting failure) $A$ process $P$ is said to be a class rewriting failure when it has the form obj $x=C$ init $Q$ in $Q^{\prime}, P \nvdash \rightarrow$ and one of the following holds:

- Undefined class name: $C=E[c]$ for some evaluation context $E$.

- Abstract class instantiation: $C=E[L]$ for some evaluation context $E$.

Definition 2 (Runtime failure) A solution $\mathcal{D} \Vdash \mathcal{P}$ is said to be a runtime failure when one of the following holds:

- Undefined object name: there is a process $\psi_{\#} x . \ell(\widetilde{u}) \in \mathcal{P}$, and $x$ is not defined in $\mathcal{D}$.

- For some $\psi_{\#} x . \ell(\widetilde{u}) \in \mathcal{P}$ and $\psi^{\prime} x_{\#} D \in \mathcal{D}$,

- Failed privacy: $\ell \in \mathcal{F}$ and $\psi^{\prime} x$ is not a prefix of $\psi$.

- Undeclared label: $\ell \notin d l(D)$.

- Arity mismatch: $\ell(\widetilde{y})$ appears in a pattern of $D$ with different arities for $\widetilde{y}$ and $\widetilde{u}$.

Definition $3 A$ solution $\mathcal{D} \Vdash \mathcal{P}$ fails either it is a runtime failure, or for some $\psi_{\#} P \in \mathcal{P}$, the process $P$ is a class rewriting failure.

\section{B Proofs for typing}

\section{B.1 Basic properties}

In the following lemmas, we let $\Delta$ range over any right hand side of a typing judgment.

Lemma 1 (Useless variable) For any judgment of the form $A \vdash \Delta$, and any name $x$ that is not free in $\Delta$ we have:

$$
A \vdash \Delta \Leftrightarrow A+A^{\prime} \vdash \Delta
$$

where $A^{\prime}$ is either $x: \sigma$ or $x: \sigma, x: \forall X . B$. 
Lemma 2 (Substitution of type variables) Let $\eta$ be a substitution on type variables. We have:

$$
A \vdash \Delta \Rightarrow \eta(A) \vdash \eta(\Delta)
$$

Given two type schemes $\forall X . \tau$ and $\forall X^{\prime} . \tau^{\prime}$, we say that $\forall X . \tau$ is more general than $\forall X^{\prime} \cdot \tau^{\prime}$ if and only if $\tau^{\prime}$ is of the form $\tau\left\{\gamma_{\alpha} / \alpha^{\alpha \in X}\right\}$, and $X^{\prime} \cap(\operatorname{ftv}(\tau) \backslash X)=\emptyset$. This notion is also lifted to sets of assumptions as follows: $A^{\prime}$ is more general than $A$ if $A$ and $A^{\prime}$ have the same domain and for each $u$ in their domain, $A^{\prime}(u)$ is more general than $A(u)$.

Lemma 3 (Generalization) If $A \vdash \Delta$ and $A^{\prime}$ is more general than $A$, then $A^{\prime} \vdash$ $\Delta$.

Lemma 4 (Substitution of a name in a term) If $A+x: \tau \vdash \Delta$ and $A \vdash u: \tau$ then $A \vdash \Delta\{u / x\}$. Similarly, if $A+x:\left[m_{i}: \widetilde{\tau}_{i}{ }^{i \in I}\right], x:\left(f_{j}: \widetilde{\tau}_{j}{ }^{j \in J}\right) \vdash \Delta,\left(A \vdash y \cdot m_{i}:\right.$ $\left.\widetilde{\tau}_{i}\right)^{i \in I}$, and $\left(A \vdash y \cdot f: \widetilde{\tau}_{j}\right)^{j \in J}$ then $A \vdash \Delta\{y / x\}$.

Lemma 5 (Substitution of a class name in a term) Let $A+c: \forall X . \zeta(\rho) B^{W} \vdash$ $\Delta$ and $A \vdash C:: \zeta(\rho) B^{W}$ and $X \subseteq \operatorname{Gen}(\rho, B, A)$. Then $A \vdash \Delta\{C / c\}$.

\section{B.2 Subject reduction for chemical reduction(Theorem 1.1)}

Proof. Given $\vdash(\mathcal{D} \Vdash \mathcal{P})$ and $\mathcal{D} \Vdash \mathcal{P} \equiv \mathcal{D}^{\prime} \Vdash \mathcal{P}^{\prime}$. We prove that $\vdash\left(\mathcal{D}^{\prime} \Vdash \mathcal{P}^{\prime}\right)$.

According to the chemical semantics with privacy in [9], there are two possible cases for the derivation of $\mathcal{D} \Vdash \mathcal{P} \equiv \mathcal{D}^{\prime} \Vdash \mathcal{P}^{\prime}$ :

1. an instance of rule Obj, followed by a sequence of Chemistry-Obj;

2. an instance of one of the rules Par, Nil, Public-Comm, Private-Comm, Red, followed by a sequence of Chemistry.

We discuss the two cases respectively.

Case 1: Given $\mathcal{D} \Vdash \psi_{\#}$ obj $x=D$ init $P$ in $Q, \mathcal{P} \equiv \mathcal{D}, \psi x_{\#} D \Vdash \psi x_{\#} P, \psi_{\#} Q, \mathcal{P}$, where $x \notin f n(\mathcal{D}) \cup f n(\mathcal{P})$. We prove that:

$$
\vdash\left(\mathcal{D} \Vdash \psi_{\#} \text { obj } x=D \text { init } P \text { in } Q, \mathcal{P}\right) \Longleftrightarrow \vdash\left(\mathcal{D}, \psi x_{\#} D \Vdash \psi x_{\#} P, \psi_{\#} Q, \mathcal{P}\right)
$$

On one hand, if $\vdash\left(\mathcal{D} \Vdash \psi_{\#}\right.$ obj $x=D$ init $P$ in $\left.Q, \mathcal{P}\right)(1)$, we obtain

$\mathrm{RR} \mathrm{n}^{\circ} 4889$ 


$$
\begin{aligned}
& \text { Chemical-Solution } \\
& A=\cup_{\varphi z \# D^{\prime} \in \mathcal{D}} A_{z} \quad\left(A^{\varphi} \backslash\{z\} \vdash D^{\prime}:: A_{z}\right)^{\varphi z \# D^{\prime} \in \mathcal{D}}(3) \\
& \frac{A^{\psi} \vdash \text { obj } x=D \text { init } P \text { in } Q \quad\left(A^{\varphi} \vdash P^{\prime}\right)^{\varphi \# P^{\prime} \in \mathcal{P}}(2)}{\vdash\left(\mathcal{D} \Vdash \psi_{\#} \text { obj } x=D \text { init } P \text { in } Q, \mathcal{P}\right)}
\end{aligned}
$$

And a derivation of $A^{\psi} \vdash$ obj $x=D$ init $P$ in $Q$ must have the form:

$$
\begin{aligned}
& \text { Object } \\
& A^{\psi} \vdash \operatorname{self}(x) D:: \zeta(\rho) B^{W}(9) \\
& A^{\psi}+x: \forall X .[\rho], x: \forall X .(B \uparrow \mathcal{F}) \vdash P(8) \\
& A^{\psi}+x: \forall X .[\rho] \vdash Q(7) \\
& \rho=B \vdash \mathcal{M}(6) \quad \operatorname{dom}(B)=\bar{W}(5) \\
& \frac{X=\operatorname{Gen}\left(\rho, B, A^{\psi}\right) \backslash \operatorname{ctv}\left(B^{W}\right)(4)}{A^{\psi} \vdash \text { obj } x=D \operatorname{init} P \operatorname{in} Q}
\end{aligned}
$$

On the other hand, if $\vdash\left(\mathcal{D}, \psi_{\#} D \Vdash x_{\#} P, \psi_{\#} Q, \mathcal{P}\right)(10)$, we obtain

$$
\begin{aligned}
& \text { Chemical-Solution } \\
& A^{\prime}=\left(\cup_{\varphi z \# D^{\prime} \in \mathcal{D}} A_{z}^{\prime}\right) \cup A_{x}^{\prime} \\
& \left(A^{\prime \varphi} \backslash\{z\} \vdash D^{\prime}:: A_{z}^{\prime}\right)^{\varphi z \# D^{\prime} \in \mathcal{D}}(14) \quad A^{\prime \psi} \backslash\{x\} \vdash D:: A_{x}^{\prime} \\
& \frac{A^{\prime \psi x} \vdash P(13) \quad A^{\prime \psi} \vdash Q(12) \quad\left(A^{\prime \varphi} \vdash P^{\prime}\right)^{\varphi \# P^{\prime} \in \mathcal{P}}(11)}{\vdash\left(\mathcal{D}, \psi x_{\#} D \Vdash \psi x_{\#} P, \psi_{\#} Q, \mathcal{P}\right)}
\end{aligned}
$$

And a derivation of $A^{\prime \psi} \backslash\{x\} \vdash D:: A_{x}^{\prime}$ must have the form:

$$
\begin{aligned}
& \text { Definition } \\
& \rho^{\prime}=B^{\prime} \uparrow \mathcal{M}(18) \quad X^{\prime}=\operatorname{Gen}\left(\rho^{\prime}, B^{\prime}, A^{\prime \psi} \backslash\{x\}\right) \backslash \operatorname{ctv}\left(B^{\prime W^{\prime}}\right)(17) \\
& \frac{A^{\prime \psi} \backslash\{x\} \vdash \operatorname{self}(x) D:: \zeta\left(\rho^{\prime}\right) B^{\prime W^{\prime}}(16) \quad \operatorname{dom}\left(B^{\prime}\right)=\overline{W^{\prime}}(15)}{A^{\prime \psi} \backslash\{x\} \vdash D:: A_{x}^{\prime}}
\end{aligned}
$$

where $A_{x}^{\prime}=x: \forall X^{\prime} \cdot\left[\rho^{\prime}\right], x: \forall X^{\prime} .\left(B^{\prime} \uparrow \mathcal{F}\right)$.

Because $x \notin f n(\mathcal{D}) \cup f n(\mathcal{P}), x$ is not bound in $A$, but in $A^{\prime}$. Therefore, if either (1) or (10) holds, we can always choose $A^{\prime}$ or $A$, such that $A^{\prime}=A+A_{x}^{\prime}$, in which $A_{x}^{\prime}$ denotes the binding for $x$ in $A^{\prime}$. As an immediate consequence, we have $A^{\prime \psi} \backslash\{x\}=A^{\psi}(19)$, where $\psi$ is any string of names of the set $N$ of object names bound in $A$.

By equation (19), and identifying $B$ with $B^{\prime}, \rho$ with $\rho^{\prime}, W$ with $W^{\prime}$, we get $(9) \equiv(16),(6) \equiv(18),(5) \equiv(15)$ and $(4) \equiv(17)$. By these equivalences, we 
have $A_{x}^{\prime}=x: \forall X .[\rho], x: \forall X .(B \nmid \mathcal{F})$. Therefore, the following three equations hold for any $\psi$ :

$$
\begin{aligned}
A^{\prime \psi} & =A^{\psi}+x: \forall X \cdot[\rho] \\
A^{\prime \psi} \backslash\{z\} & =A^{\psi} \backslash\{z\}+x: \forall X .[\rho] \\
A^{\prime \psi x} & =A^{\psi}+x: \forall X \cdot[\rho], x: \forall X .(B \nmid \mathcal{F})
\end{aligned}
$$

Now we try to show the equivalence among other premises:

- $(3) \equiv(14)$ : by equation (21) and Lemma 1 .

- $(2) \equiv(11)$ : by equation (20) and Lemma 1 .

- $(8) \equiv(13)$ : by equation $(22)$

- $(7) \equiv(12)$ : by equation $(20)$.

To conclude, if (1) holds, we take $A^{\prime}=A+x: \forall X .[\rho], x: \forall X .(B \nmid \mathcal{F})$, then we get that (10) holds, too; conversely, if (10) holds, we take $A=A^{\prime} \backslash\{x\}$ and $A_{x}^{\prime}=x: \forall X .[\rho], x: \forall X .(B \nmid \mathcal{F})$, then we get that (1) holds, too.

Case 2 The reduction is $\mathcal{D} \Vdash \mathcal{P}_{1}, \mathcal{P} \equiv \mathcal{D} \Vdash \mathcal{P}_{2}, \mathcal{P}$. There are several subcases, according to the leaf node in the derivation tree.

\section{Case Nil.}

Given $\mathcal{D} \Vdash \psi_{\#} 0, \mathcal{P} \equiv \mathcal{D} \Vdash \mathcal{P}$. We prove that $\vdash\left(\mathcal{D} \Vdash \psi_{\#} 0, \mathcal{P}\right) \Longleftrightarrow$ $\vdash(\mathcal{D} \Vdash \mathcal{P})$. Indeed the judgment $A \vdash \psi_{\# 0}$ is always true, for any environment $A$.

\section{Case Par.}

Given $\mathcal{D} \Vdash \psi_{\#}(P \& Q), \mathcal{P} \equiv \mathcal{D} \Vdash \psi_{\#} P, \psi_{\#} Q, \mathcal{P}$. Our purpose is to prove that $\vdash\left(\mathcal{D} \Vdash \psi_{\#}(P \& Q), \mathcal{P}\right) \Longleftrightarrow \vdash\left(\mathcal{D} \Vdash \psi_{\#} P, \psi_{\#} Q, \mathcal{P}\right)$. We apply rule Chemical-Solution, then it suffices to prove that the two judgments $A^{\psi} \vdash P \& Q$ and $A^{\psi} \vdash P, A^{\psi} \vdash Q$ are equivalent. This follows by rules Parallel.

\section{Case Join.}

Given $\mathcal{D} \Vdash \psi_{\#} x .\left(M \& M^{\prime}\right), \mathcal{P} \equiv \mathcal{D} \Vdash \psi_{\#} x . M, \psi_{\#} x . M^{\prime}, \mathcal{P}$. We prove that $\vdash\left(\mathcal{D} \Vdash \psi_{\#} x .\left(M \& M^{\prime}\right), \mathcal{P}\right) \Longleftrightarrow \vdash\left(\mathcal{D} \Vdash \psi_{\#} x . M, \psi_{\#} x . M^{\prime}, \mathcal{P}\right)$. This is similar to the above case, and relies on rules Chemical-Solution and Join Parallel. 


\section{Case Public-Comm.}

Given $\mathcal{D}, \psi x \# D \Vdash \psi^{\prime}{ }_{\#} x . m(\widetilde{u}), \mathcal{P} \rightarrow \mathcal{D}, \psi x_{\#} D \Vdash \psi x \# x . m(\widetilde{u}), \mathcal{P}$. We prove $\vdash\left(\mathcal{D}, \psi x_{\#} D \Vdash \psi^{\prime} \# x . m(\widetilde{u}), \mathcal{P}\right) \Longrightarrow \vdash\left(\mathcal{D}, \psi x_{\#} D \Vdash \psi x_{\#} x . m(\widetilde{u}), \mathcal{P}\right)$. Let us assume that $A^{\psi^{\prime}} \vdash x \cdot m(\widetilde{u})(23)$. We must show that $A^{\psi x} \vdash$ $x . m\left(u_{i}{ }^{i \in I}\right)$ where $\widetilde{u}=u_{i}{ }^{i \in I}$. A complete derivation of (23) must be of the form:

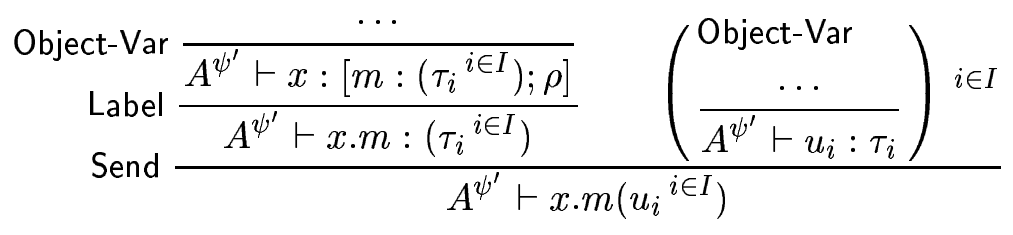

This derivation, which does not use any internal type assumption of $A$ (any Private-Message rule), is not affected by replacing $A^{\psi^{\prime}}$ with $A^{\psi x}$. Thus, $A^{\psi x} \vdash x . m\left(u_{i}{ }^{i \in I}\right)$ holds.

\section{Case Private-Comm.}

Given $\mathcal{D}, \psi x_{\#} D \Vdash \psi x \psi_{\#}^{\prime} x . f(\widetilde{u}), \mathcal{P} \rightarrow \mathcal{D}, \psi x_{\#} D \Vdash \psi x_{\#} x . f(\widetilde{u}), \mathcal{P}$. We show $\vdash\left(\mathcal{D}, \psi x_{\#} D \Vdash \psi x \psi_{\#}^{\prime} x . f(\widetilde{u}), \mathcal{P}\right) \Longrightarrow \vdash\left(\mathcal{D}, \psi x_{\#} D \Vdash \psi x_{\#} x . f(\widetilde{u}), \mathcal{P}\right)$. Let us assume that $A^{\psi x \psi^{\prime}} \vdash x . f(\widetilde{u})(24)$. We must show that $A^{\psi x} \vdash$ $x . f\left(u_{i}{ }^{i \in I}\right)$ where $\widetilde{u}=u_{i}{ }^{i \in I}$. A complete derivation of (24) must be of the form:

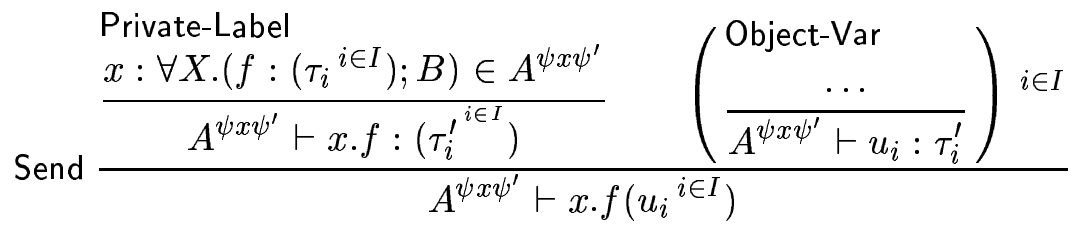

in which $\tau_{i}^{\prime}=\tau_{i}\left\{\gamma_{\alpha} / \alpha^{\alpha \in X}\right\}$.

Note that the only internal type assumption used in the premises is on $x$, which remains in $A^{\psi x}$. Thus, as in case Public-Comm, we can replace $A^{\psi x \psi^{\prime}}$ by $A^{\psi x}$ in this derivation and conclude that $A^{\psi x} \vdash x . f\left(u_{i}{ }^{i \in I}\right)$.

\section{Case Red.}

Given $\mathcal{D}, \psi_{\#} D \Vdash \psi x_{\#} x .(M \sigma), \mathcal{P} \rightarrow \mathcal{D}, \psi x_{\#} D \Vdash \psi x_{\#}(P \sigma), \mathcal{P}$, where $D$ is of the form $M \triangleright P$ or $D^{\prime}$ and $M$ is of the form $\&_{i \in I} \ell_{i}\left(\widetilde{x}_{i}\right)$. We prove $\vdash\left(\mathcal{D}, \psi_{\#} D \Vdash \psi x_{\#} x .(M \sigma), \mathcal{P}\right) \Longrightarrow \vdash\left(\mathcal{D}, \psi_{\#} D \Vdash \psi x_{\#}(P \sigma), \mathcal{P}\right)$. A derivation of $\vdash\left(\mathcal{D}, \psi x_{\#} D \Vdash \psi x \# x .(M \sigma), \mathcal{P}\right)$ must have the following 
premises, by the rule Chemical-Solution:

$$
\begin{aligned}
& A=\left(\cup_{\varphi z \# D^{\prime \prime} \in \mathcal{D}} A_{z}\right) \cup A_{x} \\
& \left(A^{\varphi} \backslash\{z\} \vdash D^{\prime \prime}:: A_{z}\right)^{\varphi z \# D^{\prime \prime} \in \mathcal{D}} \\
& A^{\psi} \backslash\{x\} \vdash D:: A_{x} \\
& A^{\psi x} \vdash x .(M \sigma) \\
& \left(A^{\varphi} \vdash P^{\prime}\right)^{\varphi \# P^{\prime} \in \mathcal{P}}
\end{aligned}
$$

To prove $\vdash\left(\mathcal{D}, \psi x_{\#} D \Vdash \psi x_{\#}(P \sigma), \mathcal{P}\right)$ it suffices to show that $A^{\psi x} \vdash P \sigma$. Note that $A^{\psi x}=A^{\psi}+A_{x}$ follows from (25).

The derivation of (27) must end with rule Definition with the premises

$$
\begin{gathered}
A_{x}=x: \forall X .[\rho], x: \forall X .(B \vdash \mathcal{F}) \\
\rho=B \nmid \mathcal{M} \\
X=\operatorname{Gen}\left(\rho, B, A^{\psi} \backslash\{x\}\right) \backslash \operatorname{ctv}\left(B^{W}\right) \\
A^{\psi} \backslash\{x\} \vdash \operatorname{self}(x) D:: \zeta(\rho) B^{W} \\
\operatorname{dom}(B)=\bar{W}
\end{gathered}
$$

On the other hand, the derivation of (33) must have a sub-derivation for the reaction $M \triangleright P$ :

$$
\begin{aligned}
& \text { Reaction } \\
& A^{\prime} \vdash M:: B_{0}(36) \\
& \frac{A^{\psi} \backslash\{x\}+x:[\rho], x:(B \nmid \mathcal{F})+A^{\prime} \vdash P(35) \quad \operatorname{dom}\left(A^{\prime}\right)=\operatorname{fn}(M)}{A^{\psi} \backslash\{x\}+x:[\rho], x:(B \nmid \mathcal{F}) \vdash M \triangleright P:: \zeta(\rho) B_{0}^{\operatorname{col}(M)}}
\end{aligned}
$$

where for every $i \in I, B_{0}\left(\ell_{i}\right)=\widetilde{\tau}_{i}(37)$.

This derivation is then followed by several Disjunction derivations and finally a Self-Binding derivation to reach (33). By the Disjunction rule, we know that $\operatorname{col}(M) \subseteq W$ (38) and $B_{0} \subseteq B$ (39). With (37) and (39), we have, $\forall i . i \in I, B\left(\ell_{i}\right)=\widetilde{\tau}_{i}(40)$

The derivation of (28) must have the form:

$$
\text { Join Parallel } \frac{\left(\begin{array}{c}
A^{\psi}+A_{x} \vdash x \cdot \ell_{i}:: \widetilde{\tau}_{i}^{\prime}(42) \\
\text { Send } \frac{A^{\psi}+A_{x} \vdash \sigma\left(\widetilde{x}_{i}\right): \widetilde{\tau}_{i}^{\prime}(41)}{A^{\psi}+A_{x} \vdash x \cdot \ell_{i}\left(\sigma\left(\widetilde{x}_{i}\right)\right)}
\end{array}\right)}{A^{\psi}+A_{x} \vdash x \cdot(M \sigma)}
$$

The judgment (42) is derived by either rule Private-Label or Label, depending on whether $\ell$ is private or public. In both cases, each tuple $\widetilde{\tau}_{i}^{\prime}$ is an

$\mathrm{RR} \mathrm{n}^{\circ} 4889$ 
instance of the generic type $\forall X . B\left(\ell_{i}\right)$, with a substitution $\eta_{i}$ of domain $X \cap \mathrm{ftv}\left(B\left(\ell_{i}\right)\right)$, namely, $\widetilde{\tau}_{i}^{\prime}=\eta_{i}\left(\widetilde{\tau}_{i}\right)(43)$. By (38) and the definitions of $\operatorname{col}(B)$ and $\operatorname{ctv}\left(B^{W}\right)$, we have $f t v\left(B\left(\ell_{i}\right)\right) \cap \mathrm{ftv}\left(B\left(\ell_{j}\right)\right) \subseteq \operatorname{ctv}\left(B^{W}\right)$, hence by (32), $f t v\left(B\left(\ell_{i}\right)\right) \cap \mathrm{ftv}\left(B\left(\ell_{j}\right)\right) \cap X=\emptyset$, for every distinct $i$ and $j$ in $I$. Thus, the sets $\left(X \cap \operatorname{ftv}\left(B\left(\ell_{i}\right)\right)\right)^{i \in I}$, i.e. $\operatorname{dom}\left(\eta_{i}\right)^{i \in I}$ form a partition of $X$. Let $\eta$ be the sum of substitutions $\oplus^{i \in I} \eta_{i}$, then we have $\widetilde{\tau}_{i}^{\prime}=\eta\left(\widetilde{\tau}_{i}\right)$ (44) from (43). Observe that the domain of $\eta$ is included in $X$ and is thus disjoint from free type variables of $A^{\psi}$.

Applying Lemma 2 to (35), we have $\eta\left(A^{\psi}+x:[\rho], x:(B \nmid \mathcal{F})\right)+\eta\left(A^{\prime}\right) \vdash P$, that is, $A^{\psi}+x:[\eta(\rho)], x: \eta(B \nmid \mathcal{F})+\eta\left(A^{\prime}\right) \vdash P$. Because $A_{x}$ is more general than $x:[\eta(\rho)], x: \eta(B \nmid \mathcal{F})$, by Lemma 3 , we get $A^{\psi}+A_{x}+\eta\left(A^{\prime}\right) \vdash P$. On the other hand, by (41) and (44), we have $A^{\psi}+A_{x} \vdash \sigma\left(\widetilde{x}_{i}\right): \eta\left(\widetilde{\tau}_{i}\right)$ for all $i \in I$, that is in fact, $\left(A^{\psi}+A_{x} \vdash \sigma\left(x_{i}\right): \eta\left(\tau_{i}\right)\right)^{x_{i}: \tau_{i} \in A^{\prime}}$. Thus using Lemma 4, we derive $A^{\psi}+A_{x} \vdash P \sigma$.

\section{B.3 Subject reductions for class rewriting (Theorem 1.2)}

We first give some useful lemmas.

Lemma 6 For any pattern $J$, we have $d l(J)=\overline{\operatorname{col}(J)}$.

Lemma 7 For any class $C$, if $A \vdash C:: \zeta(\rho) B^{W}$, then $\operatorname{dl}(C)=\operatorname{dom}(B)$.

Lemma 8 If $A \vdash W$ with $F:: W^{\prime}$, let $F=\left.\right|_{i \in I} \pi_{i} \Rightarrow W_{i}$, we have $\overline{W^{\prime}} \subseteq \bar{W} \cup$ $\left(\cup_{i \in I} \pi_{i}\right) \cup\left(\cup_{i \in I} \overline{W i}\right)$.

Proof. By induction on the depth of the derivation of $A \vdash W$ with $F:: W^{\prime}$.

Lemma 9 If $A \vdash C:: \zeta(\rho) B^{W}$, then we have $\bar{W} \subseteq \operatorname{dom}(B)$.

Proof. By induction on the depth of the derivation of $A \vdash C:: \zeta(\rho) B^{W}$ together with Lemma 8. 
Lemma 10 (Filter rewriting) If all the following conditions hold:

$$
\begin{aligned}
& C \text { with } S \rightarrow C^{\prime} \quad A \vdash C:: \zeta(\rho) B^{W} \quad A \vdash S:: B^{\prime F} \\
& \vdash W \text { with } F:: W^{\prime} \quad B \uparrow B^{\prime}
\end{aligned}
$$

then $A \vdash C^{\prime}:: \zeta(\rho)\left(\left(B^{\prime} \uparrow \overline{W^{\prime}}\right) \oplus B\right)^{W^{\prime}}$.

Proof. We reason by induction on the depth of the derivation of $C$ with $S \rightarrow C^{\prime}$.

\section{$\S 1$. Basic cases}

Case Filter-Apply. Let us assume that

$$
\begin{gathered}
K_{1} \& K \triangleright P \text { with } K_{1} \Rightarrow K_{2} \triangleright Q \mid S \\
\rightarrow K_{2} \& K \triangleright P \& Q \text { or } d l\left(K_{1}\right) \backslash \mathrm{dl}\left(K_{2}\right) \\
A \vdash K_{1} \& K \triangleright P:: \zeta(\rho) B^{W} \\
A \vdash K_{1} \Rightarrow K_{2} \triangleright Q \mid S:: B^{\prime F} \\
\vdash W \text { with } F:: W^{\prime} \\
B \uparrow B^{\prime}
\end{gathered}
$$

The judgment (2) is derived by:

$$
\begin{aligned}
& \text { Synchronization } \\
& \frac{A^{\prime} \vdash K_{1}:: B_{1}(9) \quad A^{\prime} \vdash K:: B_{0}(8)}{A^{\prime} \vdash K_{1} \& K:: B_{1} \oplus B_{0}(10)} \\
& \text { Reaction } \frac{A+A^{\prime} \vdash P(7) \quad \operatorname{dom}\left(A^{\prime}\right)=f n\left(K_{1} \& K\right)(6)}{A \vdash K_{1} \& K \triangleright P:: \zeta(\rho)\left(B_{1} \oplus B_{0}\right)^{\operatorname{col}\left(K_{1} \& K\right)}}
\end{aligned}
$$

where $B=B_{1} \oplus B_{0}(11)$ and $W=\operatorname{col}\left(K_{1} \& K\right)(12)$.

The judgment (3) is derived by:

$$
\begin{array}{ll}
\text { Modifier-Clause } & \\
A^{\prime \prime} \vdash K_{1}:: B_{1}^{\prime}(15) & A \vdash S:: B^{\prime \prime} F^{\prime} \\
A^{\prime \prime} \vdash K_{2}:: B_{2}^{\prime}(16) & \operatorname{col}\left(K_{1}\right)=\{\pi\}(13) \\
A+A^{\prime \prime} \vdash Q(17) & \operatorname{dom}\left(A^{\prime \prime}\right)=f n(K 2)(14) \\
\hline A \vdash K_{1} \Rightarrow K_{2} \triangleright Q \mid S:: B^{\prime F}
\end{array}
$$

where $B^{\prime}=B_{1}^{\prime} \oplus B_{2}^{\prime} \oplus B^{\prime \prime}(18)$ and $F=\left(\pi \Rightarrow \operatorname{col}\left(K_{2}\right)\right) \mid F^{\prime}$. 
By the definition of $\operatorname{col}\left(K_{1} \& K\right)$ together with (12) and (13), we get $W=$ $\left\{\pi \uplus \pi_{i} \mid \pi_{i} \in \operatorname{col}(K)\right\}$. A derivation of the judgment (4) has the form:

$$
\operatorname{Or} \frac{\left(\begin{array}{l}
\text { Apply } \\
A \vdash \pi \uplus \pi_{i} \text { with }\left(\pi \Rightarrow \operatorname{col}\left(K_{2}\right)\right) \mid F^{\prime}::\left\{\pi_{i} \uplus \pi_{j} \mid \pi_{j} \in \operatorname{col}\left(K_{2}\right)\right\}
\end{array}\right) \pi_{i} \in \operatorname{col}(K)}{\vdash\left\{\pi \uplus \pi_{i} \mid \pi_{i} \in \operatorname{col}(K)\right\} \text { with }\left(\pi \Rightarrow \operatorname{col}\left(K_{2}\right)\right) \mid F^{\prime}:: W^{\prime}}
$$

in which, $W^{\prime}=\cup_{\pi_{i} \in \operatorname{col}(K)}\left\{\pi_{i} \uplus \pi_{j} \mid \pi_{j} \in \operatorname{col}\left(K_{2}\right)\right\}=\operatorname{col}\left(K_{2} \& K\right)$.

Then, the following implications hold:

- $(9),(15) \Longrightarrow \operatorname{dom}\left(B_{1}\right)=\operatorname{dom}\left(B_{1}^{\prime}\right)(19)$

- (19), (11), (18), (5) $\Longrightarrow B_{1}=B_{1}^{\prime}(20)$

- $(20) \Longrightarrow A^{\prime}$ and $A^{\prime \prime}$ coincide on $f n\left(K_{1}\right)$

- (14), (15) $\Longrightarrow \mathrm{fn}\left(K_{1}\right) \subseteq \mathrm{fn}\left(K_{2}\right)(22)$

- $(22),(6),(14) \Longrightarrow$

$$
\left\{\begin{array}{l}
\operatorname{dom}\left(A^{\prime}\right) \cap \operatorname{dom}\left(A^{\prime \prime}\right)=\mathrm{fn}\left(K_{1}\right) \\
\operatorname{dom}\left(A^{\prime}\right) \cup \operatorname{dom}\left(A^{\prime \prime}\right)=\operatorname{dom}\left(A^{\prime}+A^{\prime \prime}\right)=f n\left(K_{2} \& K\right)
\end{array}\right.
$$

Following (21) and (23), and applying Lemma 1 to (8) and (16), we derive $A^{\prime}+A^{\prime \prime} \vdash K:: B_{0}(25)$ and $A^{\prime}+A^{\prime \prime} \vdash K_{2}:: B_{2}^{\prime}(26)$. Because we can always use $\alpha$-conversion to make the free names fresh, we have $\operatorname{dom}(A) \cap \operatorname{dom}\left(A^{\prime}\right)=\emptyset$ and $\operatorname{dom}(A) \cap \operatorname{dom}\left(A^{\prime \prime}\right)=\emptyset$. Then similarly, applying Lemma 1 to (7) and (17), we derive $A+A^{\prime}+A^{\prime \prime} \vdash P(27)$ and $A+A^{\prime}+A^{\prime \prime} \vdash Q$ (28). Therefore, we can build a derivation as follows:

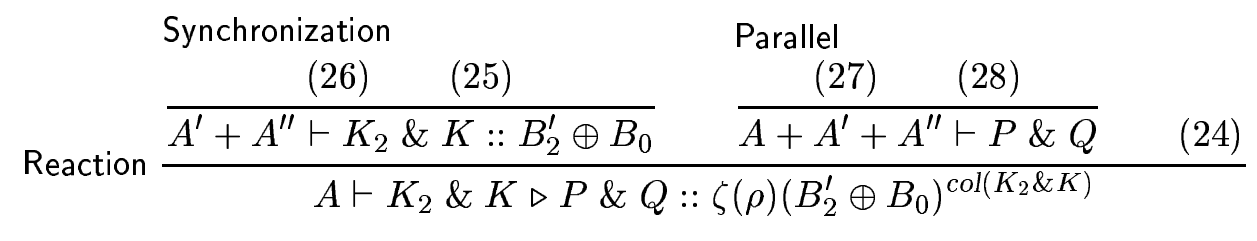

On the other hand, by rule Abstract, we can also deduce:

$$
\begin{aligned}
& \text { Abstract } \\
& \frac{\operatorname{dom}\left(B_{1}^{\prime \prime}\right)=d l\left(K_{1}\right) \backslash d l\left(K_{2}\right)}{A \vdash d l\left(K_{1}\right) \backslash d l\left(K_{2}\right):: \zeta(\rho) B_{1}^{\prime \prime}}
\end{aligned}
$$

where $B_{1}^{\prime \prime}=B_{1}^{\prime} \uparrow\left(d l\left(K_{1}\right) \backslash d l\left(K_{2}\right)\right)$. 
So by the rule Disjunction, we get

$$
A \vdash K_{2} \& K \triangleright P \& Q \text { or } d l\left(K_{1}\right) \backslash d l\left(K_{2}\right):: \zeta(\rho)\left(B_{2}^{\prime} \oplus B_{0} \oplus B_{1}^{\prime \prime}\right)^{\operatorname{col}\left(K_{2} \& K\right)}
$$

Because $\overline{W^{\prime}}=d l\left(K_{2} \& K\right)$, we get $B^{\prime} \uparrow \overline{W^{\prime}}=\left(B^{\prime} \uparrow d l\left(K_{2}\right)\right) \cup\left(B^{\prime} \uparrow d l(K)\right)$, then because $d l(K) \subseteq \operatorname{dom}(B)$ and $(5)$, we get $\left(B^{\prime} \uparrow \overline{W^{\prime}}\right) \oplus B=\left(B^{\prime} \uparrow d l\left(K_{2}\right)\right) \oplus B=$ $B_{2}^{\prime} \oplus B_{1} \oplus B_{0}=B_{2}^{\prime} \oplus B_{0} \oplus B_{1}^{\prime}$. Our goal now is to prove the following equation is true.

$$
B_{2}^{\prime} \oplus B_{0} \oplus B_{1}^{\prime}=B_{2}^{\prime} \oplus B_{0} \oplus B_{1}^{\prime \prime}
$$

And this is trivial because $B_{1}^{\prime} \uparrow\left(d l\left(K_{1}\right) \backslash d l\left(K_{2}\right)\right)=B_{1}^{\prime} \backslash d l\left(K_{2}\right)$ and $d l\left(K_{2}\right) \subseteq$ $\operatorname{dom}\left(B_{2}^{\prime}\right)$.

Case Filter-End. Let us assume

$$
\begin{gathered}
C \text { with } \emptyset \rightarrow C \\
A \vdash C:: \zeta(\rho) B^{W} \\
A \vdash \emptyset:: B^{\prime F} \\
\vdash W \text { with } F:: W^{\prime} \\
B \uparrow B^{\prime}
\end{gathered}
$$

In judgment (31), $B^{\prime}$ and $F$ must be empty because of rule Modifier-Empty. So $\left(B^{\prime} \uparrow \overline{W^{\prime}}\right) \oplus B=B$, and we have the derivation for (32) as follows:

$$
\operatorname{Or} \frac{\left(\begin{array}{l}
\text { End } \\
\vdash \pi_{i} \text { with } \emptyset::\left\{\pi_{i}\right\}
\end{array}\right) \pi_{i} \in W}{\vdash W \text { with } \emptyset:: \cup_{\pi_{i} \in W}\left\{\pi_{i}\right\}}
$$

in which, $W^{\prime}=\cup_{\pi_{i} \in W}\left\{\pi_{i}\right\}=W$. We conclude from (30) that $A \vdash C$ :: $\left(\left(B^{\prime} \uparrow \overline{W^{\prime}}\right) \oplus B\right)^{W^{\prime}}$.

Case Filter-Abstract. Let us assume

$$
\begin{gathered}
L \text { with } S \longrightarrow L \\
A \vdash L:: \zeta(\rho) B^{W} \\
A \vdash S:: B^{\prime F} \\
\vdash W \text { with } F:: W^{\prime} \\
B \uparrow B^{\prime}
\end{gathered}
$$

By rule Abstract, we know that in judgment (35), $W=\emptyset$. Then the derivation for (37) can be written as:

$$
\frac{\text { Or }}{\vdash \emptyset \text { with } F:: \emptyset}
$$

$\mathrm{RR} \mathrm{n}^{\circ} 4889$ 
which means $W^{\prime}=\emptyset=W$. So $\left(B^{\prime} \uparrow \overline{W^{\prime}}\right) \oplus B=B$. Then we reach our goal by $(35)$.

\section{§2. Inductive cases}

Case Filter-Next. Let us assume

$$
\begin{gathered}
M \triangleright P \text { with } K_{1} \Rightarrow K_{2} \triangleright Q \mid S \rightarrow C^{\prime} \\
A \vdash M \triangleright P:: \zeta(\rho) B^{W} \\
A \vdash K_{1} \Rightarrow K_{2} \triangleright Q \mid S:: B^{\prime F} \\
\vdash W \text { with } F:: W^{\prime} \\
B \uparrow B^{\prime}
\end{gathered}
$$

The judgment (39) has two premises by Filter-Next: $M \triangleright P$ with $S \rightarrow C^{\prime}$ (44) and $d l\left(K_{1}\right) \nsubseteq d l(M)$ (45). And in the judgment (40), we have $W=\operatorname{col}(M)$ (46) by rule Reaction.

A derivation of (41) is of the form:

$$
\begin{aligned}
& \text { Modifier-Clause } \\
& A^{\prime} \vdash K_{1}:: B_{1} \quad A \vdash S:: B^{\prime F^{\prime}}(47) \\
& A^{\prime} \vdash K_{2}:: B_{2} \quad \operatorname{col}\left(K_{1}\right)=\{\pi\}(48) \\
& A+A^{\prime} \vdash Q \quad \operatorname{dom}\left(A^{\prime}\right)=f n(K 2) \\
& A \vdash K_{1} \Rightarrow K_{2} \triangleright Q \mid S:: B^{\prime^{F}}
\end{aligned}
$$

where $B^{\prime}=B_{1} \oplus B_{2} \oplus B^{\prime \prime}(49)$ and $F=\left(\pi \Rightarrow \operatorname{col}\left(K_{2}\right)\right) \mid F^{\prime}$.

Applying Lemma 6 to (45) together with (46) and (48), we obtain $\pi \nsubseteq \bar{W}$, that is $\forall \pi_{i} \in W . \pi \nsubseteq \pi_{i}$. So a derivation of (42) must look like:

$$
\operatorname{Or} \frac{\left(\frac{\pi \nsubseteq \pi_{i} \quad \vdash \pi_{i} \text { with } F^{\prime}:: W_{i}(50)}{\vdash \pi_{i} \text { with } \pi \Rightarrow \operatorname{col}\left(K_{2}\right) \mid F^{\prime}:: W_{i}}\right) \pi_{i} \in W}{\vdash W \text { with } \pi \Rightarrow \operatorname{col}\left(K_{2}\right) \mid F^{\prime}:: W^{\prime}}
$$

in which, $W^{\prime}=\cup_{\pi_{i} \in W} W_{i}$. Then (50) gives us this derivation:

$$
\frac{\left(\vdash \pi_{i} \text { with } F^{\prime}:: W_{i}\right)^{\pi_{i} \in W}}{\vdash W \text { with } F^{\prime}:: W^{\prime}(51)}
$$


Moreover, judgments (43) and (49) render $B^{\prime \prime} \uparrow B$ (52). So by applying induction hypothesis to $(44),(40),(47),(51)$ and (52), we derive

$$
A \vdash C^{\prime}:: \zeta(\rho)\left(\left(B^{\prime \prime} \vdash \overline{W^{\prime}}\right) \oplus B\right)^{W^{\prime}}
$$

Because $\overline{W^{\prime}} \subseteq \operatorname{dom}\left(\left(B^{\prime \prime} \uparrow \overline{W^{\prime}}\right) \oplus B\right)$ by Lemma 9 , we have

$$
\operatorname{dom}\left(\left(B_{1} \oplus B_{2}\right) \uparrow \overline{W^{\prime}}\right) \subseteq \overline{W^{\prime}} \subseteq \operatorname{dom}\left(\left(B^{\prime \prime} \uparrow \overline{W^{\prime}}\right) \oplus B\right)(54)
$$

So we get

$$
\begin{array}{rlrl} 
& \left(B^{\prime} \uparrow \overline{W^{\prime}}\right) \oplus B & \\
= & \left(\left(B_{1} \oplus B_{2} \oplus B^{\prime \prime}\right) \uparrow \overline{W^{\prime}}\right) \oplus B & & \text { by }(49) \\
= & \left(\left(B_{1} \oplus B_{2}\right) \uparrow \overline{W^{\prime}}\right) \oplus\left(B^{\prime \prime} \uparrow \overline{W^{\prime}}\right) \oplus B & \text { " } \oplus \text { distributes over } \oplus " \\
= & \left(B^{\prime \prime} \uparrow \overline{W^{\prime}}\right) \oplus B & & \text { by }(54)
\end{array}
$$

Namely, by (53), we obtain

$$
A \vdash C^{\prime}:: \zeta(\rho)\left(\left(B^{\prime} \vdash \overline{W^{\prime}}\right) \oplus B\right)^{W^{\prime}}
$$

Case Filter-Or. Let us assume

$$
\begin{gathered}
\left(C_{1} \text { or } C_{2}\right) \text { with } S \rightarrow C_{1}^{\prime} \text { or } C_{2}^{\prime} \\
A \vdash C_{1} \text { or } C_{2}:: \zeta(\rho) B^{W} \\
A \vdash S:: B^{\prime F} \\
\vdash W \text { with } F:: W^{\prime} \\
B \uparrow B^{\prime}
\end{gathered}
$$

The judgment (55) has two premises by rule Filter-Or: $C_{1}$ with $S \rightarrow C_{1}^{\prime}(60)$ and $C_{2}$ with $S \longrightarrow C_{2}^{\prime}(61)$.

A derivation of (56) has the form:

$$
\begin{aligned}
& \text { Disjunction } \\
& \frac{A \vdash C_{1}:: \zeta(\rho) B_{1}^{W_{1}}(63) \quad A \vdash C_{2}:: \zeta(\rho) B_{2}^{W_{2}}(62)}{A \vdash C_{1} \text { or } C_{2}:: \zeta(\rho) B^{W}}
\end{aligned}
$$

where $B=B_{1} \oplus B_{2}(64)$ and $W=W_{1} \cup W_{2}$ (65). So the derivation of (58) can be written like:

$$
\frac{\stackrel{\text { Or }}{\left(\vdash W_{i} \text { with } F:: W_{i}^{\prime}(66)\right)^{i=1,2}}}{\vdash W \text { with } F:: W^{\prime}}
$$

$\mathrm{RR} \mathrm{n}^{\circ} 4889$ 
where $W^{\prime}=W_{1}^{\prime} \cup W_{2}^{\prime}$. In addition, combining (59) and (64), we get $B_{1} \uparrow$ $B^{\prime}(67)$ and $B_{2} \uparrow B^{\prime}(68)$.

Applying the induction hypothesis to (60), (63), (57), (66) and (67), we obtain

$$
A \vdash C_{1}^{\prime}:: \zeta(\rho)\left(\left(B^{\prime} \uparrow \overline{W_{1}^{\prime}}\right) \oplus B_{1}\right)^{W_{1}^{\prime}}
$$

Similarly, applying the induction hypothesis to (61), (62), (57), (66) and (68), we obtain

$$
A \vdash C_{2}^{\prime}:: \zeta(\rho)\left(\left(B^{\prime} \uparrow \overline{W_{2}^{\prime}}\right) \oplus B_{2}\right)^{W_{2}^{\prime}}
$$

Then by the Disjunction rule, we derive

$$
\begin{aligned}
& A \vdash C_{1}^{\prime} \text { or } C_{2}^{\prime}:: \zeta(\rho)\left(\left(B^{\prime} \uparrow \overline{W_{1}^{\prime}}\right) \oplus\left(B^{\prime} \uparrow \overline{W_{2}^{\prime}}\right) \oplus B_{1} \oplus B_{2}\right)^{W_{1}^{\prime} \cup W_{2}^{\prime}} \\
\equiv & A \vdash C_{1}^{\prime} \text { or } C_{2}^{\prime}:: \zeta(\rho)\left(\left(B^{\prime} \uparrow\left(\overline{W_{1}^{\prime}} \cup \overline{W_{2}^{\prime}}\right)\right) \oplus B\right)^{W_{1}^{\prime} \cup W_{2}^{\prime}} \\
\equiv & A \vdash C_{1}^{\prime} \text { or } C_{2}^{\prime}:: \zeta(\rho)\left(\left(B^{\prime} \uparrow W^{\prime}\right) \oplus B\right)^{W^{\prime}}
\end{aligned}
$$

Lemma 11 (Class reduction preserve typing) if $A+x:[\rho], x .(B \nmid \mathcal{F}) \vdash C::$ $\zeta(\rho) B^{W}$ and $C \stackrel{x}{\longrightarrow} C^{\prime}$ then $A+x:[\rho], x .(B \nmid \mathcal{F}) \vdash C^{\prime}:: \zeta(\rho) B^{W}$.

Proof. We reason by induction on the depth of the derivation of $C \stackrel{x}{\longmapsto} C^{\prime}$.

(For conciseness, we write $A_{x}$ for $A+x:[\rho], x .(B \nmid \mathcal{F})$.)

\section{§1. Basic cases}

Case Self. Let $\operatorname{self}(z) C \stackrel{x}{\rightarrow} C\{x / z\}$ and let $A_{x} \vdash \operatorname{self}(z) C:: \zeta(\rho) B^{W}$. A derivation of this judgment must end with an instance of Self-Binding. Hence,

$$
A_{x}+z:[\rho], z \cdot(B \vdash \mathcal{F}) \vdash C:: \zeta(\rho) B^{W}
$$

Then by applying Lemma 4 , we have $A_{x} \vdash C\{x / z\}:: \zeta(\rho) B^{W}$.

Case Or-Pat. Let $J_{1}$ or $J_{2} \triangleright P \stackrel{x}{\longmapsto} J_{1} \triangleright P$ or $J_{2} \triangleright P$ and let $A_{x} \vdash J_{1}$ or $J_{2} \triangleright$ $P:: \zeta(\rho) B^{W}$. The derivation of this judgment must end with the following derivation:

$$
\begin{aligned}
& \frac{A^{\prime} \vdash J_{1}:: B_{1} \quad A^{\prime} \vdash J_{2}:: B_{2}}{A^{\prime} \vdash J_{1} \text { or } J_{2}:: B} \text { Alternative } \\
& \text { Reaction } \frac{A_{x}+A^{\prime} \vdash P \quad \operatorname{dom}\left(A^{\prime}\right)=f n\left(J_{1} \text { or } J_{2}\right)}{A_{x} \vdash J_{1} \text { or } J_{2} \triangleright P:: \zeta(\rho) B^{\operatorname{col}\left(J_{1}\right) \cup \operatorname{col}\left(J_{2}\right)}}
\end{aligned}
$$


where $B$ is $B_{1} \oplus B_{2}$ and $\operatorname{col}\left(J_{1}\right) \cup \operatorname{col}\left(J_{2}\right)=W$. Since $f n\left(J_{1}\right.$ or $\left.J_{2}\right)=f n\left(J_{1}\right)=$ $\mathrm{fn}\left(J_{2}\right)$, we have:

$$
\text { Disjunction } \frac{\left(\frac{\begin{array}{l}
\text { Reaction } \\
A^{\prime} \vdash J_{i}:: B_{i} \quad A_{x}+A^{\prime} \vdash P \quad \operatorname{dom}\left(A^{\prime}\right)=f n\left(J_{i}\right)
\end{array}}{A_{x} \vdash J_{i} \triangleright P:: \zeta(\rho) B_{i}^{\operatorname{col}\left(J_{i}\right)}}\right) i=1,2}{A_{x} \vdash J_{1} \triangleright P \text { or } J_{2} \triangleright P:: \zeta(\rho) B^{\operatorname{col}\left(J_{1}\right) \cup \operatorname{col}\left(J_{2}\right)}}
$$

That is $A_{x} \vdash J_{1} \triangleright P$ or $J_{2} \triangleright P:: \zeta(\rho) B^{W}$.

Case Abstract-Cut. Let $C$ or $L \stackrel{x}{\longmapsto} C$ or $L^{\prime}$ and $A_{x} \vdash C$ or $L:: \zeta(\rho) B^{W}$ and $L^{\prime}=L \backslash d l(C)(1)$, with $L \neq L^{\prime}$. The derivation of the judgment $A_{x} \vdash C$ or $L:: \zeta(\rho) B^{W}$ must end with the following derivation:

$$
\text { Disjunction } \frac{A_{x} \vdash C:: \zeta(\rho) B_{1}^{W}}{\frac{\operatorname{Abstract}}{\operatorname{dom}\left(B_{2}\right)=L(2)}} \begin{gathered}
A_{x} \vdash L:: \zeta(\rho) B_{2}^{\emptyset} \\
\hline A_{x} \vdash C \text { or } L:: \zeta(\rho)\left(B_{1} \oplus B_{2}\right)^{W}
\end{gathered}
$$

where $B=B_{1} \oplus B_{2}$.

Applying Lemma 7 and (2) to (1), we get:

$$
\begin{aligned}
L^{\prime} & =L \backslash \operatorname{dl}(C) \\
& =L \backslash \operatorname{dom}\left(B_{1}\right) \\
& =\operatorname{dom}\left(B_{2}\right) \backslash \operatorname{dom}\left(B_{1}\right)
\end{aligned}
$$

As a consequence, we have:

$$
\begin{aligned}
& B_{1} \oplus\left(B_{2} \uparrow L^{\prime}\right) \\
= & B_{1} \oplus\left(B_{2} \uparrow\left(\operatorname{dom}\left(B_{2}\right) \backslash \operatorname{dom}\left(B_{1}\right)\right)\right) \\
= & B_{1} \oplus B_{2}
\end{aligned}
$$

Therefore we can derive:

$$
\text { Disjunction } \frac{A_{x} \vdash C:: \zeta(\rho) B_{1}^{W}}{A_{x} \vdash C \text { or } L^{\prime}:: \zeta(\rho)\left(B_{1} \oplus B_{2}\right)^{W}} \frac{\begin{array}{c}
\text { Abstract } \\
A_{x} \vdash L^{\prime}:: \zeta(\rho)\left(B_{2} \uparrow L^{\prime}\right)^{\emptyset}
\end{array}}{\operatorname{dom}\left(B_{2} \uparrow L^{\prime}\right)=L^{\prime}}
$$

$\mathrm{RR} \mathrm{n}^{\circ} 4889$ 
Case Class-Abstract. Let $C$ or $\emptyset \stackrel{x}{\longmapsto} C$ and $A_{x} \vdash C$ or $\emptyset:: \zeta(\rho) B^{W}$. The derivation of this judgment must end with rule Disjunction:

$$
\text { Disjunction } \frac{A_{x} \vdash C:: \zeta(\rho) B^{W}}{A_{x} \vdash C \text { or } \emptyset:: \zeta(\rho) B^{W}}
$$

where the judgment we have to prove, namely $A_{x} \vdash C:: \zeta(\rho) B^{W}$, appears as a premise of this derivation.

Case Match. Let $A_{x} \vdash$ match $C$ with $S$ end : $\zeta(\rho) B^{W}(3)$ and match $C$ with $S$ end $\stackrel{x}{\longmapsto} C^{\prime}$ (4) with $C$ with $S \longrightarrow C^{\prime}(5)$. We must prove that $A_{x} \vdash C^{\prime}$ : $\zeta(\rho) B^{W}(6)$.

By rule Refinement, the derivation of (3) looks like:

$$
\begin{aligned}
& \text { Refinement } \\
& A \vdash C:: \zeta(\rho) B^{\prime \prime} W^{\prime \prime}(8) \\
& A \vdash S:: B^{\prime F}(9) \\
& \vdash W^{\prime \prime} \text { with } F:: W^{\prime}(10) \quad B^{\prime \prime} \uparrow B^{\prime}(7) \\
& \hline A \vdash \text { match } C \text { with } S \text { end }:: \zeta(\rho) B^{W}
\end{aligned}
$$

where $W=W^{\prime}$ and $B=\left(B^{\prime} \uparrow \overline{W^{\prime}}\right) \oplus B^{\prime \prime}$. Applying Lemma 10 to (5), (8), (9), (10) and (7), we obtain $A \vdash C^{\prime}:: \zeta(\rho)\left(\left(B^{\prime} \vdash \overline{W^{\prime}}\right) \oplus B^{\prime \prime}\right)^{W^{\prime}}$, that is (6).

\section{§2. Inductive case}

Case Class-Context. Let $E[C] \stackrel{x}{\mapsto} E\left[C^{\prime}\right]$ because of $C \stackrel{x}{\longmapsto} C^{\prime}$ and $A_{x} \vdash E[C]::$ $\zeta(\rho) B^{W}$ (11). Then $A_{x} \vdash C:: \zeta(\rho) B^{\prime W^{\prime}}$ (12) must appear somewhere in the derivation tree of (11) . By induction hypothesis, we get $A_{x} \vdash C^{\prime}::$ $\zeta(\rho) B^{\prime W^{\prime}}$ (13). Replace (12) by (13) and all the occurrences of $C$ by $C^{\prime}$ in the derivation tree of (11), we then get a derivation tree of $A_{x} \vdash E\left[C^{\prime}\right]:: \zeta(\rho) B^{W}$.

To illustrate in a more formal way, this case is equivalent to the following statement: if $A_{x} \vdash C:: \zeta(\rho) B^{\prime W^{\prime}}$ implies $A_{x} \vdash C^{\prime}:: \zeta(\rho) B^{\prime W^{\prime}}$, and $A_{x} \vdash$ $E[C]:: \zeta(\rho) B^{W}$, we have $A_{x} \vdash E\left[C^{\prime}\right]:: \zeta(\rho) B^{W}$. We prove this by induction on the structure of $E[\cdot]$.

1. Base The base case is $E[\cdot]=[\cdot]$, it is obvious. 


\section{Induction}

- $E[\cdot]=$ match $E^{\prime}[\cdot]$ with $S$ end.

Because $A_{x} \vdash$ match $E^{\prime}[C]$ with $S$ end :: $\zeta(\rho) B^{W}$, we have the derivation

$$
\begin{aligned}
& \text { Refinement } \\
& A_{x} \vdash E^{\prime}[C]:: \zeta(\rho) B_{1}^{W_{1}}(15) \\
& A \vdash S:: B_{2}^{F}(16) \\
& \frac{\vdash W_{1} \text { with } F:: W_{2}(17) \quad B_{1} \uparrow B_{2}(14)}{A \vdash \text { match } E^{\prime}[C] \text { with } S \text { end }:: \zeta(\rho) B^{W}}
\end{aligned}
$$

where $B=\left(B_{2} \uparrow \overline{W_{2}}\right) \oplus B_{1}$ and $W=W_{2}$. By induction hypothesis with (15), we get $A_{x} \vdash E^{\prime}\left[C^{\prime}\right]:: \zeta(\rho) B_{1}^{W_{1}}$ (18). Then with (18), (16), (17) and (14) we can build a derivation for $A \vdash$ match $E^{\prime}[C]$ with $S$ end :: $\left.\zeta(\rho)\left(B_{1} \uparrow \overline{W_{2}}\right) \oplus B_{2}\right)^{W_{2}}$ by rule Refinement, that is $A_{x} \vdash E\left[C^{\prime}\right]:: \zeta(\rho) B^{W}$.

- $E[\cdot]=E^{\prime}[\cdot]$ or $C^{\prime \prime}$.

Because $A_{x} \vdash E^{\prime}[C]$ or $C^{\prime \prime}:: \zeta(\rho) B^{W}$, we have the derivation

\section{Disjunction}

$$
\frac{A_{x} \vdash E^{\prime}[C]:: \zeta(\rho) B_{1}^{W_{1}}(20) \quad A_{x} \vdash C^{\prime \prime}:: \zeta(\rho) B_{2}^{W_{2}}(19)}{A_{x} \vdash E[C] \text { or } C^{\prime \prime}:: \zeta(\rho) B^{W}}
$$

where $B=B_{1} \oplus B_{2}$ and $W=W_{1} \cup W_{2}$. Using induction hypothesis on (20), we get $A_{x} \vdash E^{\prime}\left[C^{\prime}\right]:: \zeta(\rho) B_{1}^{W_{1}}(21)$. Then with (21) and (19) we can build a derivation for $A_{x} \vdash$ $E\left[C^{\prime}\right]$ or $C^{\prime \prime}:: \zeta(\rho)\left(B_{1} \oplus B_{2}\right)^{W_{1} \cup W_{2}}$ by rule Disjunction, that is $A_{x} \vdash E\left[C^{\prime}\right]:: \zeta(\rho) B^{W}$.

- $E[\cdot]=C^{\prime \prime}$ or $E^{\prime}[\cdot]$.

Symmetrically similar to the previous case.

Now we prove the Theorem 1.2

Proof. Given $A \vdash P$ and $P \longmapsto P^{\prime}$, we prove that $A \vdash P^{\prime}$.

According to the rewriting semantics of the class language in [9], there are two cases for the derivation of $P \longmapsto P^{\prime}$. 
Case Class-Subst. Let us assume that $A \vdash$ class $c=C$ in $P(1)$ and class $c=C$ in $P \longmapsto P\{C / c\}$. The two premises: $A \vdash C:: \zeta(\rho) B^{W}(2)$ and $A+c: \forall$ Gen $(\rho, B, A) . \zeta(\rho) B^{W} \vdash P(3)$ must appear in the derivation of (1) by rule Class. Then applying Lemma 5 to (2) and (3), we get $A \vdash P\{C / c\}$.

Case Class-Red. Let $A \vdash$ obj $x=C$ init $P$ in $Q(4)$ and obj $x=C$ init $P$ in $Q \longmapsto$ obj $x=C^{\prime}$ init $P$ in $Q$, under the assumption that $C \stackrel{x}{\rightarrow} C^{\prime}(5)$.

A derivation of (4) has the shape

$$
\begin{gathered}
\frac{A+x:[\rho], x:(B \nmid \mathcal{F}) \vdash C:: \zeta(\rho) B^{W}(6)}{A \vdash \operatorname{self}(x) C:: \zeta(\rho) B^{W}} \text { Self-Binding } \\
\rho=B \nmid \mathcal{M} \quad \operatorname{dom}(B)=\bar{W} \quad X=\operatorname{Gen}(\rho, B, A) \backslash \operatorname{ctv}\left(B^{W}\right) \\
\text { Object } \frac{A+x: \forall X .[\rho], x: \forall X .(B \nmid \mathcal{F}) \vdash P \quad A+x: \forall X .[\rho] \vdash Q}{A \vdash \operatorname{obj} x=C \text { init } P \text { in } Q}
\end{gathered}
$$

Applying Lemma 11 to (5) and (6), we obtain $A+x:[\rho], x:(B \nmid \mathcal{F}) \vdash$ $C^{\prime}:: \zeta(\rho) B^{W}$, which we can substitute for $(6)$ in the previous derivation and conclude $A \vdash$ obj $x=C^{\prime}$ init $P$ in $Q$.

\section{B.4 Safety (Theorem 2)}

Proof. Let us assume that $\vdash(\mathcal{D} \Vdash \mathcal{P})$. By the rule Chemical-Solution, we have these premises true:
$\left(A^{\psi} \backslash\{x\} \vdash D:: A_{x}\right)^{\psi x \# D \in \mathcal{D}}$
$\left(A^{\psi} \vdash P\right)^{\psi \# P \in \mathcal{P}}$
$A=\cup_{\psi x \# D \in \mathcal{D}} A_{x}(3)$

We check that no class rewriting failure as defined in Definition 1 or runtime failure as defined in Definition 2 occurs.

\section{No class rewriting failures}

1. No undefined class name. Let assume that for some $\psi_{\#} P \in \mathcal{P}, P=$ (obj $x=E[c]$ init $Q$ in $Q^{\prime}$ ), and $P \nvdash \rightarrow$. Because of (2), we have $A^{\psi} \vdash$ obj $x=E[c]$ init $Q$ in $Q^{\prime}$. Following the rule Object, we must have the premise $A^{\psi} \vdash \operatorname{self}(x) E[c]:: \zeta(\rho) B^{W}$, which is the root of a derivation tree where $c: \forall X . \zeta(\rho)^{\prime} B^{\prime W^{\prime}} \in\left(A^{\psi}+x:[\rho], x:(B \uparrow \mathcal{F})\right)$ must appear as a leaf. That is to say, we get $c: \forall X . \zeta(\rho)^{\prime} B^{\prime W^{\prime}} \in A^{\psi}$. But on the other hand, by (3) we know 
that $A^{\psi}$ only has bindings for object names, which contradicts the previous fact that binding for $c$ exists in $A^{\psi}$ and $c$ is not an object name. So there cannot be a process in $\mathcal{P}$ which has an undefined class name.

2. No abstract class instantiation. Let assume that for some $\psi_{\#} P \in \mathcal{P}$, $P=\left(\right.$ obj $x=E[L]$ init $Q$ in $\left.Q^{\prime}\right)$, and $P \not \rightarrow$. According to the rewriting semantics, $P \nvdash \rightarrow$ implies $E[L] \stackrel{x}{\rightarrow}$, and then

$$
E[\cdot]=[\cdot] \mid E[\cdot] \text { or } C \mid C \text { or } E[\cdot]
$$

Let us assume $E[L]=C^{\prime}$ or $L$ (the $L$ or $C^{\prime}$ case is symmetrically similar) and $C^{\prime}$ or $L \stackrel{x}{\rightarrow}$ (4).

Because of (2), we must have a derivation for $A^{\psi} \vdash$ obj $x=\left(C^{\prime}\right.$ or $\left.L\right)$ init $Q$ in $Q^{\prime}$

$$
\begin{gathered}
\text { Self-Binding } \\
\frac{A^{\psi}+x:[\rho], x:(B \nmid \mathcal{F}) \vdash C^{\prime} \text { or } L:: \zeta(\rho) B^{W}(6)}{A^{\psi} \vdash \operatorname{self}(x)\left(C^{\prime} \text { or } L\right):: \zeta(\rho) B^{W}} \\
\rho=B \uparrow \mathcal{M} \quad \operatorname{dom}(B)=\bar{W}(5) \quad X=\operatorname{Gen}(\rho, B, A) \backslash \operatorname{ctv}\left(B^{W}\right) \\
\text { Object } \frac{A^{\psi}+x: \forall X .[\rho], x: \forall X .(B \nmid \mathcal{F}) \vdash Q \quad A^{\psi}+x: \forall X .[\rho] \vdash Q^{\prime}}{A \vdash \text { obj } x=\left(C^{\prime} \text { or } L\right) \text { init } Q \text { in } Q^{\prime}}
\end{gathered}
$$

where a derivation of (6) looks like

$$
\begin{gathered}
\text { Abstract } \\
\frac{\operatorname{dom}\left(B_{2}\right)=L(8)}{A^{\psi}+x:[\rho], x:(B \nmid \mathcal{F}) \vdash L:: \zeta(\rho) B_{2}^{\emptyset}(9)} \\
\text { Disjunction } \\
\frac{A^{\psi}+x:[\rho], x:(B \nmid \mathcal{F}) \vdash C^{\prime}:: \zeta(\rho) B_{1}^{W}(7)}{A^{\psi}+x:[\rho], x:(B \nmid \mathcal{F}) \vdash C^{\prime} \text { or } L:: \zeta(\rho) B^{W}}
\end{gathered}
$$

in which $B=B_{1} \oplus B_{2}$ and $W=W_{1}$. By (4), we have $d l\left(C^{\prime}\right) \cap L=\emptyset$. Applying Lemma 7 to (7), we get $d l\left(C^{\prime}\right)=\operatorname{dom}\left(B_{1}\right)$. Then because of (8), we get $\operatorname{dom}\left(B_{1}\right) \cap \operatorname{dom}\left(B_{2}\right)=\emptyset$. Applying Lemma 9 to $(7)$, we get $\overline{W_{1}} \subseteq \operatorname{dom}\left(B_{1}\right)$, then finally, we obtain $\overline{W_{1}} \cap \operatorname{dom}\left(B_{2}\right)=\emptyset(10)$. On the other hand, premise (5) requires $\operatorname{dom}\left(B_{1} \oplus B_{2}\right)=\overline{W_{1}}$, that is $\operatorname{dom}\left(B_{1}\right) \cup \operatorname{dom}\left(B_{2}\right)=\overline{W_{1}}$, which gives $\operatorname{dom}\left(B_{2}\right) \subseteq \overline{W_{1}}$, and contradicts (10).

To sum up, if $\vdash(\mathcal{D} \Vdash \mathcal{P})$, there cannot be a process in $\mathcal{P}$ which is a class rewriting failure.

$\mathrm{RR} \mathrm{n}^{\circ} 4889$ 


\section{No runtime failures}

1. No undefined object name. Suppose that there is a process $\psi_{\#} x . \ell\left(u_{i}{ }^{i \in I}\right) \in$ $\mathcal{P}$, such that $x$ is not defined in $\mathcal{D}$. Because of (2), we have the following derivation

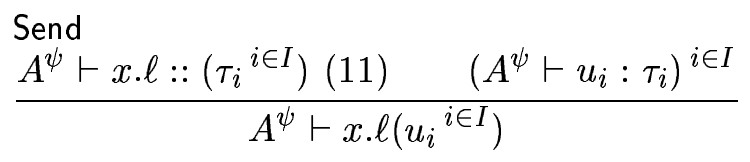

Depending on whether $\ell$ is public or private, we get from Object-Var together with Label or Private-Label that $x: \forall X . \tau \in A^{\psi}$ or $x: \forall X .(f: \widetilde{\tau} ; B) \in A^{\psi}$. On the other hand, by (3), we see that if $x$ is not defined in $\mathcal{D}, x$ will not be bound in $A$, neither in $A^{\psi}$. Contradiction.

2. No failed privacy. Suppose we have $\psi_{\#} x . f(\widetilde{u}) \in \mathcal{P}$ and $\psi^{\prime} x_{\#} D \in \mathcal{D}$, we prove that $\psi^{\prime} x$ is a prefix of $\psi$.

A derivation of $A^{\psi} \vdash x . f\left(u_{i}{ }^{i \in I}\right)$ must be:

$$
\frac{\frac{\cdots}{A^{\psi} \vdash x . f::\left(\tau_{i}{ }^{i \in I}\right)}(12) \quad\left(A^{\psi} \vdash u_{i}: \tau_{i}\right)^{i \in I}}{A^{\psi} \vdash x . f\left(u_{i}{ }^{i \in I}\right)} \text { Send }
$$

where derivation (12) is an instance of Private-Label. The premise of (12) requires that $x: \forall X .\left(f:\left(\tau_{i}{ }^{i \in I}\right) ; B\right) \in A^{\psi}$. Therefore, by definition of $A^{\psi}$, variable $x$ must appear in $\psi$. Furthermore, by well-formedness of chemical solutions, a name can have a unique prefix. Since $\psi^{\prime}$ is already a prefix of $x$, then $\psi$ must be of the form $\psi^{\prime} x \psi^{\prime \prime}$.

3. No undeclared label. Given $\psi_{\#} x . \ell(\widetilde{u}) \in \mathcal{P}$ and $\psi^{\prime} x_{\#} D \in \mathcal{D}$, we prove that $\ell \in d l(D)$.

By rule Definition, the judgment $A^{\psi^{\prime}} \backslash\{x\} \vdash D:: A_{x}$, where $A_{x}=x: \forall X$. $[\rho], x:$ $\forall X .(B \nmid \mathcal{F})$, is derived by the following premises:

$$
\begin{array}{cr}
A^{\psi^{\prime}} \backslash\{x\} \vdash \operatorname{self}(x) D:: \zeta(\rho) B^{W} & \rho=B \nmid \mathcal{M}(13) \\
X=\operatorname{Gen}\left(\rho, B, A^{\psi^{\prime}} \backslash\{x\}\right) \backslash \operatorname{ctv}\left(B^{W}\right) & \operatorname{dom}(B)=\bar{W}
\end{array}
$$

Since $A^{\psi} \vdash x \cdot \ell(\widetilde{u})$ by (2), either $\rho$ is of the form $\ell: \widetilde{\tau} ; \rho^{\prime}$ or $B$ is of the form $\ell: \widetilde{\tau} ; B^{\prime}$ depending on whether $\ell$ is public or private. Using (13), we know that in either case, $\ell$ is in $\operatorname{dom}(B)$. The conclusion follows by Lemma 7 . 
4. No arity mismatch. Suppose we have $\psi_{\#} x . \ell(\widetilde{u}) \in \mathcal{P}, \psi^{\prime} x_{\#} D \in \mathcal{D}, D=$ $(M \triangleright P)$ or $D^{\prime}$, and $M=\ell(\widetilde{y}) \& J$, we prove that $\widetilde{u}$ and $\widetilde{y}$ have the same arity. As in the previous case, we have $A^{\psi^{\prime}} \backslash\{x\} \vdash \operatorname{self}(x) D:: \zeta(\rho) B^{W}$, and a derivation of this judgment must have a leaf derivation for $M \triangleright P$

$$
\begin{aligned}
& \text { Message-Pattern } \\
& \text { Reaction } \frac{\frac{\widetilde{y}: \widetilde{\tau} \in A^{\prime}(14)}{A^{\prime} \vdash \ell(\widetilde{y})::(\ell: \widetilde{\tau})} \quad A^{\prime} \vdash J:: B^{\prime}}{A^{\prime} \vdash \ell(\widetilde{y}) \& J::(\ell: \widetilde{\tau}) \oplus B^{\prime}} \quad \ldots
\end{aligned}
$$

where $B_{1}=(\ell: \widetilde{\tau}) \oplus B^{\prime}$ and $B_{1} \subseteq B$. So we get $A^{\psi} \vdash x . \ell:: \widetilde{\tau}\left\{\gamma_{\alpha} / \alpha^{\alpha \in X}\right\}$ On the other hand, the derivation of $A^{\psi} \vdash x \cdot \ell(\widetilde{u})$ requires the premise $A^{\psi} \vdash$ $\widetilde{u}: \widetilde{\tau}\left\{\gamma_{\alpha} / \alpha^{\alpha \in X}\right\}$ (15). We conclude that $\widetilde{u}$ and $\widetilde{y}$ have the same arity by (14) and (15).

To sum up, if $\vdash(\mathcal{D} \Vdash \mathcal{P}), \mathcal{D} \Vdash \mathcal{P}$ cannot be a runtime failure. 


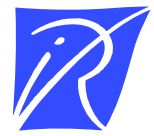

Unité de recherche INRIA Rocquencourt Domaine de Voluceau - Rocquencourt - BP 105 - 78153 Le Chesnay Cedex (France)

Unité de recherche INRIA Lorraine : LORIA, Technopôle de Nancy-Brabois - Campus scientifique 615, rue du Jardin Botanique - BP 101 - 54602 Villers-lès-Nancy Cedex (France)

Unité de recherche INRIA Rennes : IRISA, Campus universitaire de Beaulieu - 35042 Rennes Cedex (France)

Unité de recherche INRIA Rhône-Alpes : 655, avenue de l'Europe - 38330 Montbonnot-St-Martin (France)

Unité de recherche INRIA Sophia Antipolis : 2004, route des Lucioles - BP 93 - 06902 Sophia Antipolis Cedex (France)

INRIA - Domaine de Voluceau - Rocquencourt, BP 105 - 78153 Le Chesnay Cedex (France)

http://www.inria.fr

ISSN 0249-6399 